\title{
Computational capabilities of multilayer committee machines
}

\author{
J P Neirotti ${ }^{1}$ and L Franco ${ }^{2}$ \\ ${ }^{1}$ NCRG, Aston University, Birmingham, United Kingdom \\ ${ }^{2}$ Depto. de Lenguajes y Ciencias de la Computación,Universidad de Málaga, Spain \\ E-mail: j.p.neirotti@aston.ac.uk
}

\begin{abstract}
We obtained an analytical expression for the computational complexity of many layered committee machines with a finite number of hidden layers $(L<\infty)$ using the generalization complexity measure introduced by Franco et al. [1]. Although our result is valid in the large size limit and for an overlap synaptic matrix that is ultrametric, it provides a useful tool for inferring the appropriate architecture a network must have to reproduce an arbitrary realizable Boolean function.
\end{abstract}

PACS numbers: 89.70.Eg, 84.35.+i

Submitted to: J. Phys. A: Math. Gen. 


\section{Introduction}

In the present article we study the computational capabilities of a binary committee machine with an ultrametric synaptic overlap matrix. In many first-approach studies involving neural networks the single-layered perceptron has been the preferred laboratory $[2,3]$. Perceptrons are the most fundamental networks and, as it is expected, their computation capabilities are limited. The next level in terms of architectural complexity is represented by networks with at least one hidden layer and a fixed hidden to output relation. A network with an arbitrary number $K$ of units in its only hidden layer suffices to reproduce nontrivial scalar functions of $N$-dimensional variables. Exact representation of Boolean functions requires at most $K \sim O\left(2^{N}\right)$ units; continuous functions can be approximated with arbitrary accuracy if the number $K$ of units is not constrained [4]. There is an extensive number of studies done on this type of networks $[5,6,7,8]$, mostly in the area of learning theory $[9,10,11,12,13,14,15,16,17,18]$ and applications $[19,20,21,22]$. In the present paper we focus on quantifying the complexity of a binary committee machine, with no more hidden-to-input units $K$ than inputs $N$ and with a finite number of hidden layers $L$. This hardness measure, or complexity, can be used to assess what network architecture is more convenient to reproduce a given realizable Boolean function.

Attempts to quantify the complexity of an object have given origin to many formal definitions of complexity [23, 24, 25, 26, 27]. Recently [1] L. Franco proposed to quantify a function's complexity by the size of the minimal set of inputs needed to train a feed-forward network, with a predetermined architecture, until reaching zero prediction error. It was found that this minimal set is mainly formed by pairs of inputs with different classification and so adjacent to the classification boundary [28]. Further investigation showed that the average fraction of neighboring pairs with different classification (or average discrepancy) is correlated to the generalization ability of the network implementing the function, in such a way that the higher the fraction the larger the effort to reach complete generalization. They naturally concluded that the average discrepancy is a measure of how hard is to reproduce the function's behavior [29].

The average discrepancy has been used successfully for inferring the architecture of very simple machines. In [2] we used Franco's complexity measure for inferring the dilution coefficient in perceptrons; results that have been recently confirmed by other means [3]. To use Franco's complexity for inferring the suitable committee for reproducing a Boolean function we need to characterize both, the Boolean functions to be mimicked and the committees to be used. In Section 2 we present the expression for the average discrepancy for the $L+2$ layered committees. In Section 3 we study some particular cases and finite-size dependencies of the expression found in Section 2. In Section 4 we describe the Boolean functions that can be potentially described by the architectures explored in 2. In the final section we discuss our conclusions and further possible investigations. 


\section{Average discrepancy for ultrametric committees}

Let us consider a committee machine with $L$ hidden layers with $K \leq N$ hidden-to-input units. All units of all layers are binary. These links are implemented by synaptic vectors $\mathbf{w} \in \mathbb{R}^{N}$ (figure 1). The structure from the bottom up is composed by one output unit connected to $K_{L}$ units in the $L$-th hidden layer, each connected to $K_{L-1}$ units in the $(L-1)$-th level. The total number of units in the $(L-1)$-th level is then $K_{L} K_{L-1}$. Each node has an activation variable that is a function of the activation variables of the sub-tree with root at the node. Connections from units at the $\ell$-th hidden layer to units at the $\ell+1$-th layer are all set to one. To single out the variables of the $\ell$-th layer we will use the notation $\mathbf{k}_{\ell} \equiv\left[k_{L}, k_{L-1}, \ldots, k_{\ell}\right]=\mathbf{k}_{\ell+1} k_{\ell}$, which runs over all hidden units of the $\ell$-th layer. Thus

$$
\begin{aligned}
\sigma(\mathbf{S}) & \equiv \operatorname{sgn}\left(\sum_{k_{L}=1}^{K_{L}} \sigma_{k_{L}}(\mathbf{S})\right) \\
\sigma_{\mathbf{k}_{\ell}}(\mathbf{S}) & \equiv \operatorname{sgn}\left(\sum_{k_{\ell-1}=1}^{K_{\ell-1}} \sigma_{\mathbf{k}_{\ell} k_{\ell-1}}(\mathbf{S})\right) \\
\sigma_{\mathbf{k}_{1}}(\mathbf{S}) & \equiv \operatorname{sgn}\left(\frac{\mathbf{w}_{\mathbf{k}_{1}}^{\top} \mathbf{S}}{\sqrt{N}}\right),
\end{aligned}
$$

where $\mathbf{w}_{\mathbf{k}_{1}} \in \mathbb{S}^{N}$ is the synaptic vector of the $\mathbf{k}_{1}$-th unit, $\mathbf{w}^{\top}$ is the transpose of the vector $\mathbf{w}$ and $\mathbf{S} \in\{ \pm 1\}^{N}$. $\mathbb{S}^{N}$ is the surface of the $N$-hypersphere of radius $\sqrt{N}$ and $\{ \pm 1\}^{N}$ is the $N$-hypercube centered at 0 . The committee has been constructed drawing vectors from a suitable distribution over $\mathbb{S}^{N}$ such that

$$
[\mathbf{Q}]_{\mathbf{k}_{1}, \mathbf{k}_{1}^{\prime}} \equiv \frac{\mathbf{w}_{\mathbf{k}_{1}}^{\top} \mathbf{w}_{\mathbf{k}_{1}^{\prime}}}{N}=\delta_{\mathbf{k}_{1} \mathbf{k}_{1}^{\prime}}\left(1-\tilde{\zeta}_{1}\right)+\ldots+\delta_{\mathbf{k}_{L} \mathbf{k}_{L}^{\prime}}\left(\tilde{\zeta}_{L-1}-\tilde{\zeta}_{L}\right)+\tilde{\zeta}_{L}
$$

where $\tilde{\zeta}_{\ell}$ is the overlap between synaptic vectors belonging to units that have a common root in the $\ell$-th hidden layer (see figure 1) and $\delta_{\mathbf{k}_{\ell} \mathbf{k}_{\ell}^{\prime}} \equiv \prod_{m=\ell}^{L} \delta_{k_{m} k_{m}^{\prime}}$ and $\delta_{i j}=1$ if and only if $i=j$ and 0 otherwise. The structure of this matrix is block-diagonal and resembles the matrices used to represent inter-replica interactions [30].

We suppose that the elements of the overlap matrix have a natural scaling relationship with the size of the system. The argument that gives support to this conjecture runs as follows: suppose we want to draw $K \leq N$ vectors from a uniform distribution over $\mathbb{S}^{N}$, with the constraint that for any pair of vectors $\mathbf{w}_{i}$ and $\mathbf{w}_{j}$ it is satisfied that $\mathbf{w}_{i}^{\top} \mathbf{w}_{j}=N \cos (\alpha)$ for all $i \neq j$ and fixed $\alpha$. The first vector $\mathbf{w}_{1}$ drawn can be any vector on the surface of the sphere. The second vector $\mathbf{w}_{2}$ is one of the vectors located on the hyper-ring with center in $\mathbf{w}_{1}$ and radius $\sqrt{N} \sin (\alpha)$. Thus the probability $\mathcal{P}\left(\mathbf{w}_{2} \mid \mathbf{w}_{1}\right)$ has to be proportional to the volume of this hyper-ring, i.e., $\mathcal{P}\left(\mathbf{w}_{2} \mid \mathbf{w}_{1}\right) \propto$ $\sin ^{N-2}(\alpha)$. The third vector $\mathbf{w}_{3}$ has to sustain the same angle with the other two vectors, therefore the probability has to have a second factor such that $\mathcal{P}\left(\mathbf{w}_{3} \mid \mathbf{w}_{1}, \mathbf{w}_{2}\right) \mathcal{P}\left(\mathbf{w}_{2} \mid \mathbf{w}_{1}\right) \propto$ $\sin ^{N-2}(\alpha) \sin ^{N-3}(\alpha)$. After drawing $K$ vectors we have that the probability of the set of vectors is $\mathcal{P}\left(\mathbf{w}_{K} \mid \mathbf{w}_{1}, \ldots, \mathbf{w}_{K-1}\right) \mathcal{P}\left(\mathbf{w}_{K-1} \mid \mathbf{w}_{1}, \ldots, \mathbf{w}_{K-2}\right) \ldots \mathcal{P}\left(\mathbf{w}_{2} \mid \mathbf{w}_{1}\right) \propto$ 
$\sin ^{N-2}(\alpha) \sin ^{N-3}(\alpha) \ldots \sin ^{N-K}(\alpha)$. The expected overlap is then

$$
\begin{aligned}
\langle\text { overlap }\rangle & \equiv \frac{\int_{0}^{\pi / 2} \mathrm{~d} \alpha \cos (\alpha) \sin ^{z}(\alpha)}{\int_{0}^{\pi / 2} \mathrm{~d} \alpha \sin ^{z}(\alpha)}=\frac{1}{\sqrt{\pi}} \frac{\Gamma\left(\frac{2+z}{2}\right)}{\Gamma\left(\frac{3+z}{2}\right)} \\
& \simeq \sqrt{\frac{2}{\pi z}}+O\left(z^{-1}\right),
\end{aligned}
$$

with an asymptotic variance of

$$
\operatorname{var}(\text { overlap }) \simeq \frac{\pi-2}{\pi z}+O\left(z^{-1}\right)
$$

where $z=N-2+N-3+\ldots+N-K=\frac{1}{2}(K-1)[2 N-K-2]$. If $1 \geq \kappa \equiv K / N$ then

$$
\langle\text { overlap }\rangle \sim \sqrt{\frac{4 \kappa}{\pi(2-\kappa)}} \frac{1}{K}=\frac{\zeta_{1}}{K},
$$

where $\zeta_{1} \sim O(1)$. Let us suppose now that from the $K=K_{1} K_{2}$ vectors we make $K_{2}$ groups of $K_{1}$ vectors each, in such a way that the inter-group and extra-group overlaps are fixed to $\tilde{\zeta}_{1}$ and $\tilde{\zeta}_{2}$ respectively. We have proven that the inter-group overlap must scale with the inverse of the group size, i.e., $\tilde{\zeta}_{1}=\zeta_{1} / K_{1}$ and we know that there are a total of $K_{1} K_{2}$ inter-group overlaps and $K_{1} K_{2}\left(K_{1} K_{2}-1\right) / 2$ extra-group vector overlaps. A crude estimate of the average overlap is then

$$
\begin{aligned}
\langle\text { overlap }\rangle & \sim\left(\begin{array}{c}
K_{1} K_{2}+1 \\
2
\end{array}\right)^{-1}\left[\left(\begin{array}{c}
K_{1} K_{2} \\
1
\end{array}\right) \frac{\zeta_{1}}{K_{1}}+\left(\begin{array}{c}
K_{1} K_{2} \\
2
\end{array}\right) \tilde{\zeta}_{2}\right] \\
& \sim \tilde{\zeta}_{2}+O\left(1 / K_{1}^{2} K_{2}\right)
\end{aligned}
$$

and, according to (3), we must have $\tilde{\zeta}_{2} \sim O\left(1 / K_{1} K_{2}\right)$.

If the above argument is iterated $L$ times we recover the matrix defined in (2) with elements satisfying the scaling relationship:

$$
\tilde{\zeta}_{\ell}=\frac{\zeta_{\ell}}{\prod_{j=1}^{\ell} K_{j}},
$$

where $\zeta_{j} \sim O(1)$ and $\prod_{j=1}^{L} K_{j}=K$.

Observe that the matrix $\mathbf{Q}$ has the following properties

(i) $\mathbf{Q}$ is symmetric, i.e., $[\mathbf{Q}]_{\mathbf{k}_{1}, \mathbf{k}_{1}^{\prime}}=[\mathbf{Q}]_{\mathbf{k}_{1}^{\prime}, \mathbf{k}_{1}}$ for all paths $\mathbf{k}_{1}$ and $\mathbf{k}_{1}^{\prime}$

(ii) $\mathbf{Q}$ only has non-negative entries, i.e., $[\mathbf{Q}]_{\mathbf{k}_{1}, \mathbf{k}_{1}^{\prime}}>0$ for all paths $\mathbf{k}_{1}$ and $\mathbf{k}_{1}^{\prime}$

(iii) $[\mathbf{Q}]_{\mathbf{k}_{1}, \mathbf{k}_{1}^{\prime}} \geq \min \left\{[\mathbf{Q}]_{\mathbf{k}_{1}, \mathbf{k}_{1}^{\prime \prime}},[\mathbf{Q}]_{\mathbf{k}_{1}^{\prime}, \mathbf{k}_{1}^{\prime \prime}}\right\}$ for all paths $\mathbf{k}_{1}, \mathbf{k}_{1}^{\prime}$ and $\mathbf{k}_{1}^{\prime \prime}$

(iv) $1=[\mathbf{Q}]_{\mathbf{k}_{1}, \mathbf{k}_{1}} \geq \max \left\{[\mathbf{Q}]_{\mathbf{k}_{1}, \mathbf{k}_{1}^{\prime}} \forall \mathbf{k}_{1}^{\prime} \neq \mathbf{k}_{1}\right\}$,

therefore the matrix $\mathbf{Q}$ is ultrametric. Given that the overlap matrix $\mathbf{Q}$ is ultrametric we dubbed these networks ultrametric committee machines. 
The average discrepancy $[1,2]$ is defined as

$$
\mathfrak{d}_{P}^{(N)}(\mathbf{Q})=\sum_{\mathbf{S}, \mathbf{S}^{\prime} \in\{ \pm 1\}^{N}} \mathcal{P}\left(\mathbf{S}, \mathbf{S}^{\prime}\right) \frac{1-\sigma(\mathbf{S}) \sigma\left(\mathbf{S}^{\prime}\right)}{2},
$$

where $\mathbf{S}$ and $\mathbf{S}^{\prime}$ differ in exactly $P$ entries. We assume that the components of the input vector $\mathbf{S}$ are i.i.d. variables, therefore $\mathcal{P}(\mathbf{S})=\prod_{\ell=1}^{N} \mathcal{P}\left(S_{\ell}\right)$ and $\mathcal{P}(S=1)=\mathcal{P}(S=$ $-1)=\frac{1}{2}$. The input vector $\mathbf{S}^{\prime}$ is constructed by flipping $P$ entries randomly chosen from S. Thus $\mathcal{P}\left(\mathbf{S}^{\prime} \mid \mathbf{S}\right)=\left(\begin{array}{l}N \\ P\end{array}\right)^{-1} \delta_{N-\mathbf{S} \cdot \mathbf{S}^{\prime}, 2 P}$.

Following the developments of Appendix A and considering the large system-size limit $P, K \leq N \rightarrow \infty$ with $p \equiv P / N$ fixed, and by defining

$$
\begin{aligned}
& \mathcal{G}_{1}(p) \equiv 1-\frac{2}{\pi} \arccos (1-2 p) \\
& \gamma_{\ell} \equiv\left(\frac{2}{\pi}\right)^{\ell} \zeta_{\ell}\left[1+\sum_{j=1}^{\ell}\left(\frac{2}{\pi}\right)^{j} \zeta_{j}\right]^{-1} \\
& \mathcal{G}_{\ell+1}(p) \equiv 1-\frac{2}{\pi} \arccos \left(\left(1-\gamma_{\ell}\right) \mathcal{G}_{\ell}(p)+\gamma_{\ell}(1-2 p)\right)
\end{aligned}
$$

we obtain the following expression

$$
\mathfrak{d}(p, \mathbf{Q})=\frac{1}{\pi} \arccos \left(\left(1-\gamma_{L}\right) \mathcal{G}_{L}(p)+\gamma_{L}(1-2 p)\right) .
$$

\section{Particular cases and finite-size dependencies}

If we have that $\zeta_{\ell}=0$ for all $\ell=1, \ldots, L$, the matrix $\mathbf{Q}$ is the identity and the committee has a perfect tree structure. Let us introduce the notation

$$
\llbracket f \rrbracket^{k+1}(x)=\llbracket f \rrbracket^{k} \circ f(x)
$$

where $f \circ g(x)=f(g(x))$. Therefore, if we define $\psi(p) \equiv \arccos (1-2 p) / \pi$, then

$$
\mathfrak{d}(p, \mathbf{I})=\llbracket \psi \rrbracket^{L+1}(p)
$$

in agreement with [31] and references therein. Examples of curves obtained by applying (6) for tree committee machines are given in figure 2.

If the overlap of the $\ell_{0}$-th layer is too large, i.e., $\lim _{K_{1}, \ldots, K_{\ell_{0}} \rightarrow \infty}\left(\prod_{\ell=1}^{\ell_{0}} K_{\ell}\right) \tilde{\zeta}_{\ell_{0}}$ diverges, we have that

$$
\gamma_{\ell}=\left\{\begin{array}{lr}
\left(\frac{2}{\pi}\right)^{\ell} \zeta_{\ell}\left[1+\sum_{j=1}^{\ell}\left(\frac{2}{\pi}\right)^{j} \zeta_{j}\right]^{-1} & \forall \ell<\ell_{0} \\
1 & \ell=\ell_{0} \\
0 & \text { otherwise }
\end{array}\right.
$$

which immediately implies that

$$
\lim _{\zeta_{\ell_{0}} \uparrow \infty} \mathfrak{d}(p, \mathbf{Q})=\llbracket \psi \rrbracket^{L-\ell_{0}+1}(p) .
$$




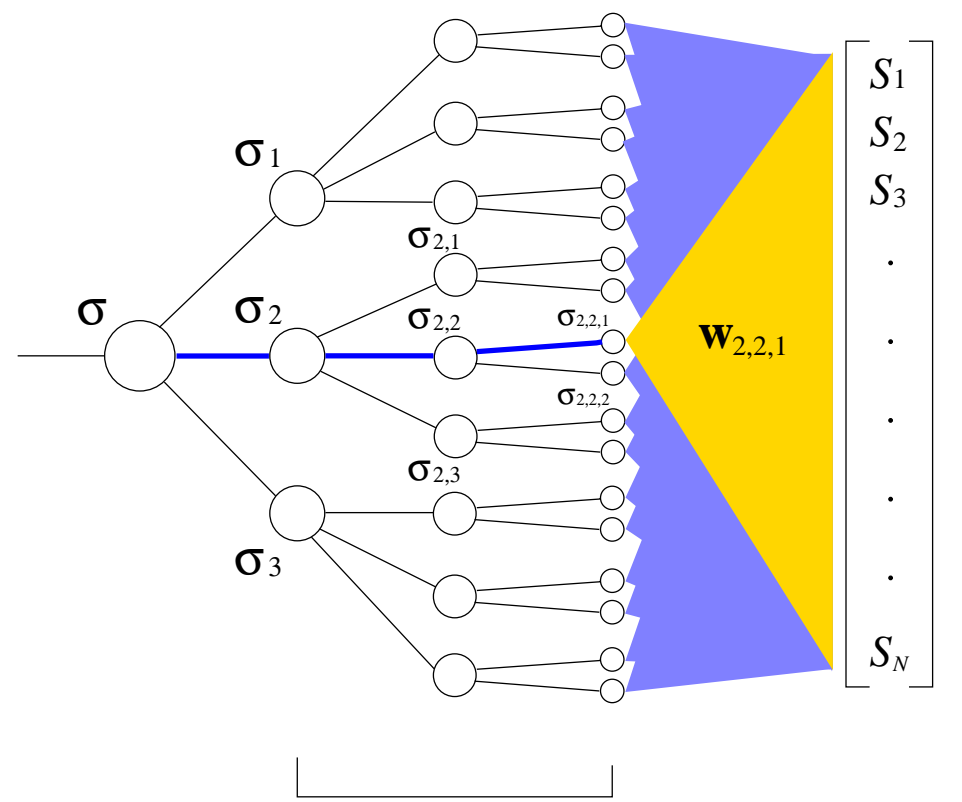

$L$ (3) hidden layers

Figure 1. Typical feed-forward network architecture studied in the present paper. This committee has $L=3$ hidden layers with synaptic overlaps in the first hidden to input layer. All the synaptic weights linking hidden to hidden and hidden to output units are set to one. Observe that the highlighted synaptic vector $\mathbf{w}_{2,2,1}$ corresponds to the output to the input layer path $\mathbf{k}_{1}=(2,2,1)$, i.e., second unit of the third hidden layer, second unit second hidden layer, first unit first hidden layer (color on-line).

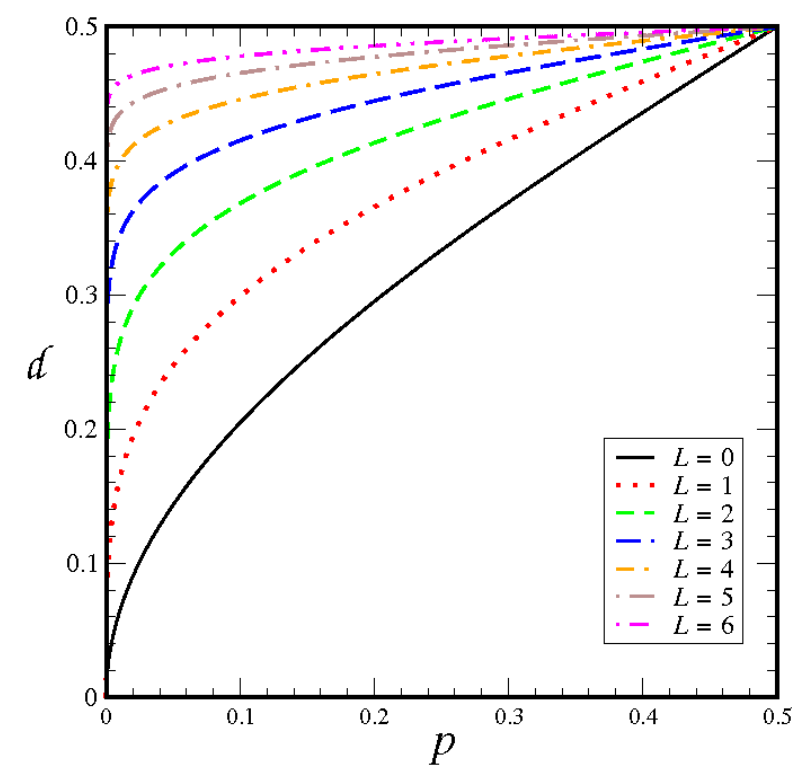

Figure 2. Discrepancy as a function of $p$ for tree committee machines with $L=0, \ldots, 6$ hidden layers, as obtained by applying (6) (color on-line). 
Therefore, a too large an overlap reduces the computational capabilities of the network by effectively deleting the first $\ell_{0}$ hidden layers.

Equation (6) is valid in the limit $P, K \leq N \rightarrow \infty$ keeping $P / N=p$ fixed. It is important to note that none of the ratios $K_{i} / N$ are relevant to this formulation. Therefore, whatever the path taken to increase the number of hidden units, the asymptotic value of the discrepancy is always the same.

In order to check this statement we run a number of numerical experiments in networks with $L=1,2$. Firstly we developed an algorithm for generating the set of synaptic vectors satisfying the relationship (2) which has the following general form:

(i) Generate $K=\prod_{l=1}^{L} K_{l}$ orthonormal vectors characterized by a suitable set of indexes $\left.\mid j_{1}, \ldots j_{L}\right) \in \mathbb{S}$ (the Gram-Schmidt algorithm may be used), with $1 \leq$ $j_{l} \leq K_{l}$ and where $\mathbb{S}$ is a vector space over $\mathbb{R}$ with an inner product such that

$$
\left(j_{1}^{\prime}, \ldots, j_{L}^{\prime} \mid j_{1}, \ldots j_{L}\right)=\prod_{l=1}^{L} \delta_{j_{l}^{\prime}, j_{l}}
$$

where $\delta_{j, k}$ is the Kronecker's delta (equal to 1 if $j=k$ and zero otherwise).

(ii) Define the block average vectors as

$$
\left.\left.\mid \mu_{l}, j_{l+1}, \ldots j_{L}\right) \equiv \frac{1}{\sqrt{K_{1}}} \sum_{j_{1}=1}^{K_{1}} \ldots \frac{1}{\sqrt{K_{l}}} \sum_{j_{l}=1}^{K_{l}} \mid j_{1}, \ldots j_{L}\right) .
$$

(iii) Define the synaptic vectors as

$$
\left.\left.\mathbf{w}_{\mathbf{j}_{1}}=\mathbf{w}_{j_{1}, \ldots, j_{L}} \equiv \sqrt{N}\left[a_{0} \mid j_{1}, \ldots j_{L}\right)+\sum_{l=1}^{L} a_{l} \mid \mu_{l}, j_{l+1}, \ldots j_{L}\right)\right],
$$

where the real coefficients $a_{l}, 0 \leq l \leq L$ are determined from the equations satisfying the definition of the matrix $\mathbf{Q}(2)$.

In the present application we have to solve the following equations for the coefficients:

$$
\begin{aligned}
\tilde{\zeta}_{1} & =\frac{2 a_{0} a_{1}}{\sqrt{K_{1}}}+a_{1}^{2} \\
1 & =\tilde{\zeta}_{1}+a_{0}^{2}
\end{aligned}
$$

for $L=1$ and

$$
\begin{aligned}
& \tilde{\zeta}_{2}=\frac{2 a_{0} a_{2}}{\sqrt{K_{1} K_{2}}}+\frac{2 a_{1} a_{2}}{\sqrt{K_{2}}}+a_{2}^{2} \\
& \tilde{\zeta}_{1}=\tilde{\zeta}_{2}+\frac{2 a_{0} a_{1}}{\sqrt{K_{1}}}+a_{1}^{2} \\
& 1=\tilde{\zeta}_{1}+a_{0}^{2}
\end{aligned}
$$

for $L=2$. 
By constructing networks like the one depicted in figure 1 with output $\sigma$ given by (1a) and synaptic vectors chosen according to the algorithm described, we estimate the average discrepancy by computing

$$
\mathfrak{d}_{P}^{(N)}[\sigma] \simeq \frac{1}{M} \sum_{m=1}^{M} \frac{1-\sigma\left(\mathbf{S}_{m}\right) \sigma\left(\mathbf{S}_{m}^{\prime}\right)}{2}
$$

over $M=10^{4}$ pairs of inputs $\left(\mathbf{S}_{m}, \mathbf{S}_{m}^{\prime}\right)$ differing in $P \approx 0.03 N$ bits. For $L=1$ we worked with networks having $K=N=279,465,651$ and 837 and overlap $\zeta_{1}=1$. The results (empty circles) are plot in figure 3 (a). The error bars have been obtained by repeating the experiment ten times and computing the variance of the averages. All the results obtained are indistinguishable with the value obtained by applying equation (6) with $\zeta_{1}=1$ and $p=0.03$, represented as a full circle.

For $L=2$ we perform two experiments, with sampling size of $10^{4}$. The first with $\zeta_{1}=\zeta_{2}=1$ and $K_{1}=K_{2}=\sqrt{N}$ for $N=625,729,841$ and 961. The results are represented by empty circles in figure 3 (b). The error bars were computed as in the previous experiment. The second experiment was ran with $\zeta_{1}=\zeta_{2}=1$ and $25=K_{2}<K_{1}=27,29$ and 31 and $N=729,841$ and 961 respectively (empty squares). Results with equal $N$ have been found indistinguishable. Moreover, a linear regression produces an extrapolated value of the discrepancy of $0.260 \pm 0.004$ that is indistinguishable to 0.2617 , the one obtained by applying (6) with $\zeta_{1}=\zeta_{2}=1$ and $p=0.03$, (full circle).

\section{Boolean functions with continuous discrepancies}

In this section we will study the Boolean functions that can be implemented by the the networks presented in figure 1. Expression (6) provides a family of curves that densely cover the set of possible discrepancy values. It is clear from $(1 b)$ that any function approached by these networks must be odd, i.e., $f(\mathbf{S})=-f(-\mathbf{S})$. It is also clear that (6) is continuous in $p$, thus we expect the discrepancy functional to be also continuous, i.e.,

$$
\forall 0 \leq P<\infty \text { and } 0<\varepsilon \in \mathbb{R}, \exists N_{0} \in \mathbb{N} /\left|\mathfrak{d}_{P}^{\left(N_{0}\right)}[f]-\mathfrak{d}_{P+1}^{\left(N_{0}\right)}[f]\right|<\varepsilon .
$$

In order to analyze what type of functions satisfy (7) we will use the decomposition of a function into an orthogonal basis set. The main development of this section is based on Ref. [32].

Let $f$ and $g:\{ \pm 1\}^{N} \rightarrow \mathbb{R}$. Consider the inner product defined by

$$
\langle f \mid g\rangle \equiv \frac{1}{2^{N}} \sum_{\{\mathbf{S}\}} f(\mathbf{S}) g(\mathbf{S})
$$

and consider also the set of parity functions $\chi_{\mathbb{I}}(\mathbf{S}) \equiv \prod_{k \in \mathbb{I}} S_{k}$ where $\mathbb{I} \subseteq[N]=$ $\{1,2, \ldots, N\}$. Observe that

$$
\left\langle\chi_{\mathbb{I}} \mid \chi_{\mathbb{I}}\right\rangle=\frac{1}{2^{N}} \sum_{\{\mathbf{S}\}} \prod_{k \in \mathbb{I} \triangle \mathbb{J}} S_{k}=\delta_{\mathbb{I}, \mathbb{J}}
$$



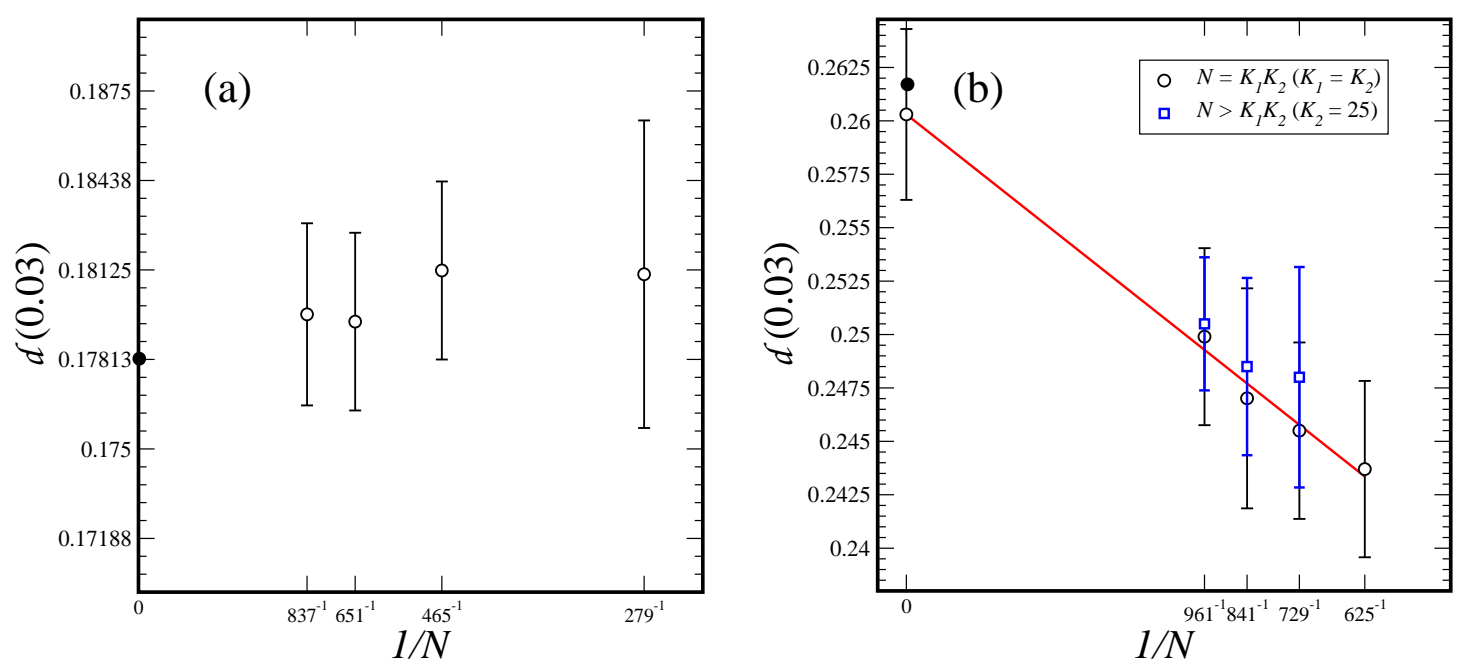

Figure 3. Finite-size dependencies of the average discrepancy at $p \approx 0.03$, measured over $M=10^{4}$ pairs. For $L=1$ we worked with networks sizes of $K=N=$ $279,465,651$ and 837 and overlap $\zeta_{1}=1$. The results (empty circles) are plot in panel (a). The error bars have been obtained by repeating the experiment ten times and computing the variance of the averages. All the results obtained are indistinguishable with the value obtained by applying equation (6) with $\zeta_{1}=1$ and $p=0.03$, represented as a full circle. For $L=2$ we perform two experiments, panel (b). We firstly considered networks with $\zeta_{1}=\zeta_{2}=1$ and $K_{1}=K_{2}=\sqrt{N}$ for $N=625,729,841$ and 961. The results are represented by empty circles and the error bars were computed as in the previous experiment. The second experiment was ran with $\zeta_{1}=\zeta_{2}=1$ and sizes of $25=K_{2}<K_{1}=27,29$ and 31 and $N=729,841$ and 961 respectively (empty squares). Results with equal $N$ have been found to be indistinguishable. Moreover, a linear regression produces an extrapolated value of the discrepancy of $0.260 \pm 0.004$ that is indistinguishable to 0.2617 , the one obtained by applying (6) with $\zeta_{1}=\zeta_{2}=1$ and $p=0.03$ (full circle).

where $\mathbb{I} \triangle \mathbb{J} \equiv \mathbb{I} \cup \mathbb{J} / \mathbb{I} \cap \mathbb{J}=\{k \in[N] \mid k \in \mathbb{I}$ xor $k \in \mathbb{J}\}$ and $\delta_{\mathbb{I}, \mathbb{J}}=1$ if both sets are equal (i.e., $\mathbb{I} \subset \mathbb{I}$ and $\mathbb{I} \subset \mathbb{I}$ ) and 0 otherwise. Given a Boolean function $f:\{ \pm 1\}^{N} \rightarrow\{ \pm 1\}$ we define the Fourier amplitudes as $\hat{f}_{\mathbb{I}} \equiv\left\langle f \mid \chi_{\mathbb{I}}\right\rangle$. It is straightforward to prove that $1=\sum_{\mathbb{I}} \hat{f}_{\mathbb{I}}^{2}$. The Fourier spectrum of $f$ is defined as the set

$$
\mathscr{S}[f] \equiv\left\{\hat{f}_{\mathbb{I}} \mid \mathbb{I} \subseteq[N]\right\}
$$

By following the developments of Appendix D and considering the definition

$$
\mathscr{A}_{r} \equiv \sum_{\mathbb{I}_{r}} \hat{f}_{\mathbb{I}_{r}}^{2}
$$

where the sum is over all the sets of indexes $\mathbb{I}_{r}$ with exactly $r$ elements, we have that the Fourier components of the function must satisfy the following condition

$$
\forall j \in \mathbb{N} \lim _{N \rightarrow \infty} \frac{1}{N^{j}} \sum_{r=1}^{N} \mathscr{A}_{r} r^{j}=0 .
$$

Thus the functions that can be approached by committees like the one depicted in figure 1 must have a Fourier spectrum satisfying the conditions elicited by (10). Observe 


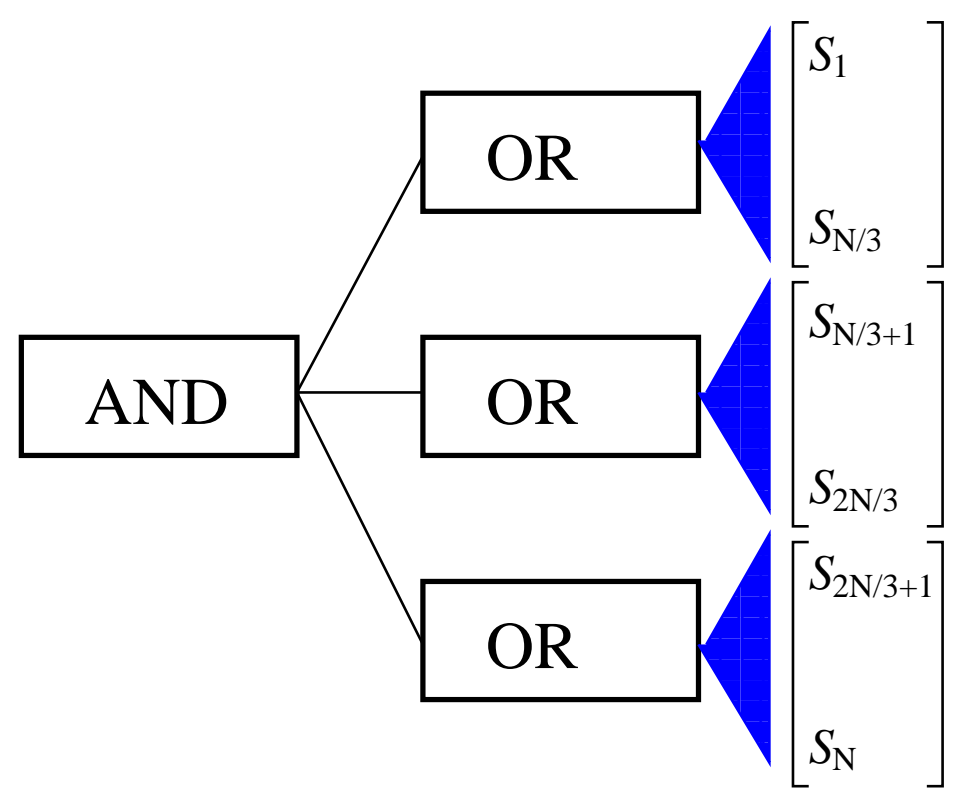

Figure 4. Graphic representation of the function $\operatorname{TRIBE}_{3}(\mathbf{S})$

that the functions not satisfying this conditions are those with Fourier components corresponding to parity functions constructed using a macroscopic (i.e., $O(N)$ ) number of bits. Trivially, the full parity machine $\chi_{[N]}$ does not satisfy (10) and its discrepancy curve is, in the large $N$ limit, discontinuous everywhere.

Consider now the function $\operatorname{TRIBE}_{n}(\mathbf{S})$ which is defined by applying the AND function to the output of $n$ OR functions, which in turn are applied to $n$ equal and disjoint segments of the input vector $\mathbf{S}$ (see figure 4). Although the functions $\operatorname{TRIBE}_{n}(\mathbf{S})$ are not odd they satisfy (10) (its Fourier spectrum is reported in [31]); they can be easily constructed from logical gates and they have also been used in studies of noise sensitivity [31, 33]. We are currently working on developing methods for approximating $\mathrm{TRIBE}_{n}$ functions with ultrametric committees.

\section{Conclusions}

The most important result of the present article is expression (6). This is the expression of the average discrepancy of a binary committee machine with $L+2$ layers as a function of the overlap parameters $\zeta_{1}, \ldots, \zeta_{L}$. It is clear that, for a fixed value of $p=P / N$, by varying these parameters the committee's average discrepancy may take any possible value between the discrepancy of a simple perceptron, i.e., $\frac{1}{\pi} \arccos (1-2 p)$, and $\frac{1}{2}$. Moreover, for a fixed value of $p$ the larger the $L$ and the smaller the overlap parameters the larger the discrepancy. This suggests that the smaller the overlap parameters and for large networks the larger the ability of the committee to compute Boolean functions with a continuous discrepancy curve.

Most results in committee machines $[14,17,18]$ are obtained with a finite number 
of hidden units. In our case we consider the regime with both, large number of input units and large number of hidden units. Although the average discrepancy appears to be a quantity that develops finite-size dependencies, expression (6) represents a good approach even for the smallest system size studied in this article.

Observe also that the relative errors in our numerical calculations are of the order of the $2 \%$. We intend to continue the study of these finite-size effects in order to obtain scaling laws that may be useful for computing the properties of finite systems. These proposed studies are limited by the number of hidden layers considered, given that the more hidden layers the larger the number of parameters $\left(K_{1}, \ldots, K_{L}\right)$ we need to increment to infinity.

\section{Appendix A. Calculation of (6)}

By using the definition (1b) and the properties of the Heaviside function we can see that

$$
\begin{aligned}
\Theta( \pm \sigma(\mathbf{S})) & =\Theta\left( \pm \sum_{k_{L}=1}^{K_{L}} \sigma_{k_{L}}(\mathbf{S})\right) \\
& =\sum_{\tau_{1}= \pm 1} \ldots \sum_{\tau_{K_{L}}= \pm 1} \Theta\left( \pm \sum_{k_{L}=1}^{K_{L}} \tau_{k_{L}}\right) \prod_{k_{L}=1}^{K_{L}} \Theta\left(\tau_{k_{L}} \sigma_{k_{L}}(\mathbf{S})\right) \\
& =\sum_{\left\{\boldsymbol{\tau}_{L}\right\}} \Theta\left( \pm \sum_{k_{L}=1}^{K_{L}} \tau_{k_{L}}\right) \prod_{k_{L}=1}^{K_{L}} \Theta\left(\tau_{k_{L}} \sigma_{k_{L}}(\mathbf{S})\right)
\end{aligned}
$$

where $\boldsymbol{\tau}_{L}=\left(\tau_{1}, \ldots, \tau_{K_{L}}\right)$ are binary variables used to represent the internal state of the hidden units in the $L$-th layer and $\sum_{\left\{\boldsymbol{\tau}_{L}\right\}} \equiv \sum_{\tau_{1}= \pm 1} \cdots \sum_{\tau_{K_{L}}= \pm 1}$. Applying this identity again we obtain

$$
\begin{gathered}
\Theta( \pm \sigma(\mathbf{S}))=\sum_{\left\{\boldsymbol{\tau}_{L}\right\}} \Theta\left( \pm \sum_{k_{L}=1}^{K_{L}} \tau_{k_{L}}\right) \prod_{k_{L}=1}^{K_{L}} \sum_{\left\{\boldsymbol{\tau}_{L-1}^{k_{L}}\right\}} \Theta\left(\tau_{k_{L}} \sum_{k_{L-1}=1}^{K_{L-1}} \tau_{k_{L}, k_{L-1}}\right) \\
\prod_{k_{L-1}=1}^{K_{L-1}} \Theta\left(\tau_{k_{L}, k_{L-1}} \sigma_{k_{L}, k_{L-1}}(\mathbf{S})\right)
\end{gathered}
$$

where $\boldsymbol{\tau}_{L-1}^{k_{L}} \equiv\left(\tau_{k_{L}, 1}, \tau_{k_{L}, 2}, \ldots, \tau_{k_{L}, K_{L-1}}\right)$ is the set of binary variables used to represent the state of the hidden units at the $L-1$-th layer, with root at the $k_{L}$ unit in the $L$-th layer. If we use the vectorial notation for the indexes, we can define the vector $\boldsymbol{\tau}_{\ell-1}^{\mathbf{k}_{\ell}} \equiv\left(\tau_{\mathbf{k}_{\ell}, 1}, \tau_{\mathbf{k}_{\ell}, 2}, \ldots, \tau_{\mathbf{k}_{\ell}, K_{\ell-1}}\right)$ as the vector where the component $\tau_{\mathbf{k}_{\ell}, m}$ represents the state of the $m$-th unit at the $\ell$ - 1 -th layer with root at the $\mathbf{k}_{\ell}$-th unit in the $\ell$-th layer. In this way we obtain that

$$
\Theta( \pm \sigma(\mathbf{S}))=\sum_{\left\{\boldsymbol{\tau}_{L}\right\}} \Theta\left( \pm \sum_{k_{L}=1}^{K_{L}} \tau_{k_{L}}\right) \prod_{k_{L}=1}^{K_{L}} \prod_{\ell=1}^{L-1} \sum_{\left\{\boldsymbol{\tau}_{L-\ell}^{\left.\mathbf{k}_{L-\ell+1}\right\}}\right.} \Theta\left(\tau_{\mathbf{k}_{L-\ell+1}} \sum_{k_{L-\ell}=1}^{K_{L-\ell}} \tau_{\mathbf{k}_{L-\ell+1}, k_{L-\ell}}\right)
$$




$$
\prod_{k_{L-\ell}=1}^{K_{L-\ell}} \prod_{k_{1}=1}^{K_{1}} \Theta\left(\tau_{\mathbf{k}_{1}} \frac{\mathbf{w}_{\mathbf{k}_{1}}^{\top} \mathbf{S}}{\sqrt{N}}\right) .
$$

We can represent the Heaviside function by using the Fourier transform of the delta function

$\Theta(x)=\int_{0}^{\infty} \mathrm{d} \eta \delta(\eta-x)=\int_{0}^{\infty} \mathrm{d} \eta \int_{-\infty}^{\infty} \frac{\mathrm{d} \hat{\eta}}{2 \pi} \exp [-i \hat{\eta}(\eta-x)]=\int \mathcal{D}(\eta, \hat{\eta}) \exp (i \hat{\eta} x)$,

where we have defined the notation $\int \mathcal{D}(x, \hat{x}) \equiv \int_{0}^{\infty} \mathrm{d} x \int_{-\infty}^{\infty} \mathrm{d} \hat{x} \exp (-i x \hat{x}) / 2 \pi$. Applying these expressions we have that, by redistributing the products,

$$
\begin{aligned}
& \Theta( \pm \sigma(\mathbf{S}))=\int \mathcal{D}\left(\eta_{L+1}, \hat{\eta}_{L+1}\right) \sum_{\left\{\boldsymbol{\tau}_{L}\right\}} \exp \left( \pm i \hat{\eta}_{L+1} \sum_{k_{L}=1}^{K_{L}} \tau_{k_{L}}\right) \\
& \times \int\left[\prod_{k_{L}=1}^{K_{L}} \mathcal{D}\left(\eta_{k_{L}}, \hat{\eta}_{k_{L}}\right)\right]\left(\prod_{k_{L}=1}^{K_{L}} \sum_{\left\{\boldsymbol{\tau}_{L-1}^{\left.\mathbf{k}_{L}\right\}}\right.}\right) \exp \left(i \sum_{k_{L}=1}^{K_{L}} \hat{\eta}_{k_{L}} \tau_{k_{L}} \sum_{k_{L-1}=1}^{K_{L-1}} \tau_{k_{L}, k_{L-1}}\right) \\
& \times \int\left[\prod_{\mathbf{k}_{L-1}} \mathcal{D}\left(\eta_{\mathbf{k}_{L-1}}, \hat{\eta}_{\mathbf{k}_{L-1}}\right)\right]\left(\prod_{\mathbf{k}_{L-1}} \sum_{\left\{\boldsymbol{\tau}_{L-2}\right\}} \exp \left(i \sum_{\mathbf{k}_{L-1}} \hat{\eta}_{\mathbf{k}_{L-1} \tau_{\mathbf{k}_{L-1}}} \sum_{k_{L-2}=1}^{K_{L-2}} \tau_{\mathbf{k}_{L-1}, k_{L-2}}\right) \ldots\right. \\
& \times \int\left[\prod_{\mathbf{k}_{2}} \mathcal{D}\left(\eta_{\mathbf{k}_{2}}, \hat{\eta}_{\mathbf{k}_{2}}\right)\right]\left(\prod_{\mathbf{k}_{2}} \sum_{\left\{\boldsymbol{\tau}_{1}^{\mathbf{k}_{2}}\right\}}\right) \exp \left(i \sum_{\mathbf{k}_{2}} \hat{\eta}_{\mathbf{k}_{2}} \tau_{\mathbf{k}_{2}} \sum_{k_{1}=1}^{K_{1}} \tau_{\mathbf{k}_{2}, k_{1}}\right) \\
& \times \int\left[\prod_{\mathbf{k}_{1}} \mathcal{D}\left(\eta_{\mathbf{k}_{1}}, \hat{\eta}_{\mathbf{k}_{1}}\right)\right] \exp \left(i \sum_{\mathbf{k}_{1}} \hat{\eta}_{\mathbf{k}_{1}} \tau_{\mathbf{k}_{1}} \frac{\mathbf{w}_{\mathbf{k}_{1}}^{\top} \mathbf{S}}{\sqrt{N}}\right),
\end{aligned}
$$

where $\prod_{\mathbf{k}_{\ell}} \equiv \prod_{k_{L}=1}^{K_{L}} \prod_{k_{L-1}=1}^{K_{L-1}} \cdots \prod_{k_{\ell}=1}^{K_{\ell}}$ and $\sum_{\mathbf{k}_{\ell}} \equiv \sum_{k_{L}=1}^{K_{L}} \sum_{k_{L-1}=1}^{K_{L-1}} \ldots \sum_{k_{\ell}=1}^{K_{\ell}}$. Observe that $\prod_{\mathbf{k}_{\ell+1}} \sum_{\left\{\boldsymbol{\tau}_{\ell} \mathbf{k}_{\ell+1}\right\}}$ is the trace over all the internal representations at the $\ell$ th layer, therefore, if we define the vector $\boldsymbol{\tau}_{\ell}$ such that $\left[\boldsymbol{\tau}_{\ell}\right]_{\mathbf{k}_{\ell}}=\tau_{\mathbf{k}_{\ell}}$ we can rewrite the trace as $\sum_{\left\{\boldsymbol{\tau}_{\ell}\right\}} \equiv \prod_{\mathbf{k}_{\ell+1}} \sum_{\left\{\boldsymbol{\tau}_{\ell} \mathbf{k}_{\ell+1}\right\}}$. Equivalently we can redefine the differentials such that $\mathcal{D}\left(\boldsymbol{\eta}_{\ell}, \hat{\boldsymbol{\eta}}_{\ell}\right) \equiv \prod_{\mathbf{k}_{\ell}} \mathcal{D}\left(\eta_{\mathbf{k}_{\ell}}, \hat{\eta}_{\mathbf{k}_{\ell}}\right)$. Finally we want to remark that $\sum_{\mathbf{k}_{\ell+1}} \hat{\eta}_{\mathbf{k}_{\ell+1}} \tau_{\mathbf{k}_{\ell+1}} \sum_{k_{\ell}=1}^{K_{\ell}} \tau_{\mathbf{k}_{\ell+1}, k_{\ell}}=\sum_{\mathbf{k}_{\ell}} \hat{\eta}_{\mathbf{k}_{\ell+1}} \tau_{\mathbf{k}_{\ell+1}} \tau_{\mathbf{k}_{\ell}}$. With all things considered and defining the variable $\tau_{k_{L+1}} \equiv \tau_{L+1}= \pm 1$ according to the prefactor of the argument, we can write

$$
\begin{aligned}
& \Theta( \pm \sigma(\mathbf{S}))=\sum_{\left\{\tau_{L+1}\right\}} \delta_{\tau_{L+1}, \pm 1} \int \mathcal{D}\left(\eta_{L+1}, \hat{\eta}_{L+1}\right) \\
& \quad \times \prod_{\ell=L}^{1} \int \mathcal{D}\left(\boldsymbol{\eta}_{\ell}, \hat{\boldsymbol{\eta}}_{\ell}\right) \sum_{\left\{\boldsymbol{\tau}_{\ell}\right\}} \exp \left(i \sum_{\mathbf{k}_{\ell}} \hat{\eta}_{\mathbf{k}_{\ell+1}} \tau_{\mathbf{k}_{\ell+1}} \tau_{\mathbf{k}_{\ell}}\right) \exp \left(i \sum_{\mathbf{k}_{1}} \hat{\eta}_{\mathbf{k}_{1}} \tau_{\mathbf{k}_{1}} \frac{\mathbf{w}_{\mathbf{k}_{1}}^{\top} \mathbf{S}}{\sqrt{N}}\right) .
\end{aligned}
$$

As it is presented in [2] we can define the sets of indexes $\mathbb{I}_{P}$ such that for each set $\mathbb{I}_{P}$ there is a vector $\mathbf{S}_{\mathbb{I}_{P}}$ such that $\left[\mathbf{S}_{\mathbb{I}_{P}}\right]_{i}=-S_{i} \forall i \in \mathbb{I}_{P}$ and $S_{i}$ otherwise. We can write 
the discrepancy as

$$
\begin{aligned}
& \mathfrak{d}_{P}^{(N)}(\mathbf{Q})=\frac{1}{2^{N}} \sum_{\{\mathbf{S}\}}\left(\begin{array}{c}
N \\
P
\end{array}\right)^{-1} \sum_{\mathbb{I}_{P}}\left[\Theta(-\sigma(\mathbf{S})) \Theta\left(\sigma\left(\mathbf{S}_{\mathbb{I}_{P}}\right)\right)+\Theta(\sigma(\mathbf{S})) \Theta\left(-\sigma\left(\mathbf{S}_{\mathbb{I}_{P}}\right)\right)\right] \\
& =\frac{1}{2^{N}} \sum_{\{\mathbf{S}\}}\left(\begin{array}{c}
N \\
P
\end{array}\right)^{-1} \sum_{\mathbb{I}_{P}} 2 \Theta(-\sigma(\mathbf{S})) \Theta\left(\sigma\left(\mathbf{S}_{\mathbb{I}_{P}}\right)\right) \\
& \times 2 \sum_{\left\{\tau_{L+1}, \bar{\tau}_{L+1}\right\}} \delta_{\tau_{L+1},-1} \delta_{\bar{\tau}_{L+1}, 1} \int \mathcal{D}\left(\eta_{L+1}, \hat{\eta}_{L+1}\right) \int \mathcal{D}\left(\eta_{L+1}^{\prime}, \hat{\eta}_{L+1}^{\prime}\right) \\
& \times \prod_{\ell=L}^{1} \int \mathcal{D}\left(\boldsymbol{\eta}_{\ell}, \hat{\boldsymbol{\eta}}_{\ell}\right) \mathcal{D}\left(\boldsymbol{\eta}_{\ell}^{\prime}, \hat{\boldsymbol{\eta}}_{\ell}^{\prime}\right) \sum_{\left\{\boldsymbol{\tau}_{\ell}, \overline{\boldsymbol{\tau}}_{\ell}\right\}} \exp \left[i \sum_{\mathbf{k}_{\ell}}\left(\hat{\eta}_{\mathbf{k}_{\ell+1}} \tau_{\mathbf{k}_{\ell+1}} \tau_{\mathbf{k}_{\ell}}+\hat{\eta}_{\mathbf{k}_{\ell+1}}^{\prime} \bar{\tau}_{\mathbf{k}_{\ell+1}} \bar{\tau}_{\mathbf{k}_{\ell}}\right)\right] \\
& \times \frac{1}{2^{N}} \sum_{\mathbf{S} \in \mathbf{Z}^{N}}\left(\begin{array}{l}
N \\
P
\end{array}\right)^{-1} \sum_{\mathbb{I}_{P}} \exp \left[i \sum_{\mathbf{k}_{1}} \frac{\mathbf{w}_{\mathbf{k}_{1}}^{\top}}{\sqrt{N}}\left(\hat{\eta}_{\mathbf{k}_{1}} \tau_{\mathbf{k}_{1}} \mathbf{S}+\hat{\eta}_{\mathbf{k}_{1}}^{\prime} \bar{\tau}_{\mathbf{k}_{1}} \mathbf{S}_{\mathbb{I}_{P}}\right)\right] .
\end{aligned}
$$

Observing that $\mathbf{w}^{\top} \mathbf{S}_{\mathbb{I}_{P}}=\mathbf{w}^{\top} \mathbf{S}-2 \sum_{j \in \mathbb{I}_{P}} w_{j} S_{j}$, we can write

$\kappa_{P, N}\left(\boldsymbol{v}_{1}, \mathbf{S}\right) \equiv \exp \left\{i \sum_{\mathbf{k}_{1}}\left[\left(\hat{\eta}_{\mathbf{k}_{1}} \tau_{\mathbf{k}_{1}}+\hat{\eta}_{\mathbf{k}_{1}}^{\prime} \bar{\tau}_{\mathbf{k}_{1}}\right) \sum_{j=1}^{N} \frac{w_{\mathbf{k}_{1}, j} S_{j}}{\sqrt{N}}-2 \hat{\eta}_{\mathbf{k}_{1}}^{\prime} \bar{\tau}_{\mathbf{k}_{1}} \sum_{j \in \mathbb{I}_{p}} \frac{w_{\mathbf{k}_{1}, j} S_{j}}{\sqrt{N}}\right]\right\}$

where $\boldsymbol{v}_{1}$ represent the set of variables in the first hidden and input layers.

The dependency on $\mathbf{S}$ can be eliminated by averaging over all possible inputs

$$
\begin{aligned}
& \frac{1}{2^{N}} \sum_{\{\mathbf{S}\}} \kappa_{P, N}\left(\boldsymbol{v}_{1}, \mathbf{S}\right)= \prod_{j \in \mathbb{I}_{P}} \cos \left(\sum_{\mathbf{k}_{1}}\left(\hat{\eta}_{\mathbf{k}_{1}} \tau_{\mathbf{k}_{1}}-\hat{\eta}_{\mathbf{k}_{1}}^{\prime} \bar{\tau}_{\mathbf{k}_{1}}\right) \frac{w_{\mathbf{k}_{1}, j}}{\sqrt{N}}\right) \\
& \times \prod_{j \notin \mathbb{I}_{P}} \cos \left(\sum_{\mathbf{k}_{1}}\left(\hat{\eta}_{\mathbf{k}_{1}} \tau_{\mathbf{k}_{1}}+\hat{\eta}_{\mathbf{k}_{1}}^{\prime} \bar{\tau}_{\mathbf{k}_{1}}\right) \frac{w_{\mathbf{k}_{1}, j}}{\sqrt{N}}\right) \\
& \simeq \exp \left[-\frac{1}{2} \sum_{\mathbf{k}_{1}, \mathbf{m}_{1}}\left(\left(\hat{\eta}_{\mathbf{k}_{1}} \tau_{\mathbf{k}_{1}}+\hat{\eta}_{\mathbf{k}_{1}}^{\prime} \bar{\tau}_{\mathbf{k}_{1}}\right) \frac{\mathbf{w}_{\mathbf{k}_{1}}^{\top} \mathbf{w}_{\mathbf{m}_{1}}}{N}\left(\hat{\eta}_{\mathbf{m}_{1}} \tau_{\mathbf{m}_{1}}+\hat{\eta}_{\mathbf{m}_{1}}^{\prime} \bar{\tau}_{\mathbf{m}_{1}}\right)\right.\right. \\
&\left.\left.-4 \hat{\eta}_{\mathbf{k}_{1}}^{\prime} \bar{\tau}_{\mathbf{k}_{1}} \sum_{j \in \mathbb{I}_{P}} \frac{w_{\mathbf{k}_{1}, j} w_{\mathbf{m}_{1}, j}}{N} \hat{\eta}_{\mathbf{m}_{1}}^{\prime} \bar{\tau}_{\mathbf{m}_{1}}\right)\right],
\end{aligned}
$$

for large enough $N$. By using the definition (2) and the result of Appendix Appendix B we have that in the thermodynamic limit $P \leq N \rightarrow \infty$ and $P / N=p$

$$
\begin{aligned}
\kappa_{p}\left(\boldsymbol{v}_{1}\right) \equiv \lim _{P \leq N \rightarrow \infty}\left(\begin{array}{c}
N \\
P
\end{array}\right)^{-1} \sum_{\mathbb{I}_{P}} \frac{1}{2^{N}} \sum_{\{\mathbf{S}\}} \kappa_{P, N}\left(\boldsymbol{v}_{1}, \mathbf{S}\right) \\
\simeq \exp \left\{-\frac{1}{2}\left(\left[\hat{\boldsymbol{\eta}}_{1} \boldsymbol{\tau}_{1}\right]-(1-2 p)\left[\hat{\boldsymbol{\eta}}_{1}^{\prime} \overline{\boldsymbol{\tau}}_{1}\right]\right)^{\top} \mathbf{Q}\left(\left[\hat{\boldsymbol{\eta}}_{1} \boldsymbol{\tau}_{1}\right]-(1-2 p)\left[\hat{\boldsymbol{\eta}}_{1}^{\prime} \overline{\boldsymbol{\tau}}_{1}\right]\right)\right. \\
\left.-2 p(1-p)\left[\hat{\boldsymbol{\eta}}_{1}^{\prime} \overline{\boldsymbol{\tau}}_{1}\right]^{\top} \mathbf{Q}\left[\hat{\boldsymbol{\eta}}_{1}^{\prime} \overline{\boldsymbol{\tau}}_{1}\right]\right\},
\end{aligned}
$$

where $\left[\hat{\boldsymbol{\eta}}_{1} \boldsymbol{\tau}_{1}\right]_{\mathbf{k}_{1}}=\hat{\eta}_{\mathbf{k}_{1}} \tau_{\mathbf{k}_{1}},\left[\hat{\boldsymbol{\eta}}_{1}^{\prime} \overline{\boldsymbol{\tau}}_{1}\right]_{\mathbf{k}_{1}}=\hat{\eta}_{\mathbf{k}_{1}}^{\prime} \bar{\tau}_{\mathbf{k}_{1}}$. 
By the use of the Hubbard-Stratonovitch identity, we can simplify this expression. Observe that

$$
\exp \left[-\frac{\phi_{\ell}^{2}}{2} \sum_{\mathbf{k}_{\ell}}\left(\sum_{k_{\ell-1}} \ldots \sum_{k_{1}} v_{\mathbf{k}_{1}}\right)^{2}\right]=\int \mathcal{D} \boldsymbol{x}_{\ell} \exp \left(i \phi_{\ell} \sum_{\mathbf{k}_{1}} x_{\mathbf{k}_{\ell}} v_{\mathbf{k}_{1}}\right)
$$

where $\phi_{\ell} \equiv \sqrt{\tilde{\zeta}_{\ell-1}-\tilde{\zeta}_{\ell}}, \phi_{1} \equiv \sqrt{1-\tilde{\zeta}_{1}}, \phi_{L+1} \equiv \sqrt{\tilde{\zeta}_{L}}, \mathcal{D} x \equiv \mathrm{d} x \exp \left(-x^{2} / 2\right) / \sqrt{2 \pi}$ and $\mathcal{D} \boldsymbol{x}_{\ell} \equiv \prod_{\mathbf{k}_{\ell}} \mathcal{D} x_{\mathbf{k}_{\ell}}$. Therefore

$\exp \left(-\frac{1}{2} \boldsymbol{v}_{1}^{\top} \mathbf{Q} \boldsymbol{v}_{1}\right)=\exp \left(-\frac{\phi_{1}^{2}}{2} \boldsymbol{v}_{1}^{\top} \boldsymbol{v}_{1}\right) \int\left(\prod_{\ell=2}^{L+1} \mathcal{D} \boldsymbol{x}_{\ell}\right) \exp \left(i \sum_{\mathbf{k}_{1}} \bar{X}_{\left[\mathbf{k}_{2}\right]} v_{\mathbf{k}_{1}}\right)$,

where $\bar{X}_{\left[\mathbf{k}_{2}\right]} \equiv \sum_{\ell=2}^{L+1} \phi_{\ell} x_{\mathbf{k}_{\ell}}$ and $x_{\mathbf{k}_{L+1}} \equiv x_{L+1}$. Given that we have two quadratic terms in the exponential of (A.3) we will need two sets of integrals. Thus

$$
\begin{aligned}
\kappa_{p}\left(\boldsymbol{v}_{1}\right)=\exp \left\{-\frac{\phi_{1}^{2}}{2}\left[\left(\left[\hat{\boldsymbol{\eta}}_{1} \boldsymbol{\tau}_{1}\right]+(1-2 p)\left[\hat{\boldsymbol{\eta}}_{1}^{\prime} \overline{\boldsymbol{\tau}}_{1}\right]\right)^{\boldsymbol{\top}}\left(\left[\hat{\boldsymbol{\eta}}_{1} \boldsymbol{\tau}_{1}\right]+(1-2 p)\left[\hat{\boldsymbol{\eta}}_{1}^{\prime} \overline{\boldsymbol{\tau}}_{1}\right]\right)\right.\right. \\
\left.\left.+4 p(1-p)\left[\hat{\boldsymbol{\eta}}_{1}^{\prime} \overline{\boldsymbol{\tau}}_{1}\right]^{\top}\left[\hat{\boldsymbol{\eta}}_{1}^{\prime} \overline{\boldsymbol{\tau}}_{1}\right]\right]\right\} \\
\times \int\left[\prod_{\ell=2}^{L+1} \mathcal{D} \boldsymbol{x}_{\ell} \mathcal{D} \boldsymbol{x}_{\ell}^{\prime}\right] \exp \left[i \sum_{\mathbf{k}_{1}} \bar{X}_{\left[\mathbf{k}_{2}\right]}\left(\hat{\eta}_{\mathbf{k}_{1}} \tau_{\mathbf{k}_{1}}+(1-2 p) \hat{\eta}_{\mathbf{k}_{1}}^{\prime} \bar{\tau}_{\mathbf{k}_{1}}\right)\right] \\
\times \exp \left[i \sqrt{4 p(1-p)} \sum_{\mathbf{k}_{1}} \bar{X}_{\left[\mathbf{k}_{2}\right]}^{\prime} \hat{\eta}_{\mathbf{k}_{1}}^{\prime} \bar{\tau}_{\mathbf{k}_{1}}\right] .
\end{aligned}
$$

We can integrate over $\mathcal{D}\left(\boldsymbol{\eta}_{1}, \hat{\boldsymbol{\eta}}_{1}\right)$ and $\mathcal{D}\left(\boldsymbol{\eta}_{1}^{\prime}, \hat{\boldsymbol{\eta}}_{1}^{\prime}\right)$

$$
\begin{aligned}
\int \mathcal{D} & \left(\boldsymbol{\eta}_{1}, \hat{\boldsymbol{\eta}}_{1}\right) \mathcal{D}\left(\boldsymbol{\eta}_{1}^{\prime}, \hat{\boldsymbol{\eta}}_{1}^{\prime}\right) \kappa_{p}\left(\boldsymbol{v}_{1}\right)= \\
& \prod_{\ell=2}^{L+1} \int \mathcal{D} \boldsymbol{x}_{\ell} \mathcal{D} \boldsymbol{x}_{\ell}^{\prime} \prod_{\mathbf{k}_{1}} \int_{0}^{\infty} \frac{\mathrm{d} \eta}{\sqrt{2 \pi}} \exp \left[-\frac{1}{2}\left(\eta-\tau_{\mathbf{k}_{1}} X_{\left[\mathbf{k}_{2}\right]}\right)^{2}\right] \mathcal{H}\left(\bar{\tau}_{\mathbf{k}_{1}}\left(\beta(p) \tau_{\mathbf{k}_{1}} \eta+X_{\left[\mathbf{k}_{2}\right]}^{\prime}\right)\right),
\end{aligned}
$$

where $X_{\left[\mathbf{k}_{2}\right]} \equiv \bar{X}_{\left[\mathbf{k}_{2}\right]} / \phi_{1}, \beta(p) \equiv(1-2 p) / \sqrt{4 p(1-p)}$ and $\mathcal{H}(x) \equiv \int_{x}^{\infty} \mathcal{D} y$ is Gardner's error function.

Finally

$$
\begin{aligned}
& \kappa_{p}\left(\boldsymbol{v}_{2}\right) \equiv \sum_{\left\{\boldsymbol{\tau}_{1}, \overline{\boldsymbol{\tau}}_{1}\right\}} \exp \left[i\left(\left[\hat{\boldsymbol{\eta}}_{2} \boldsymbol{\tau}_{2}\right]^{\top} \boldsymbol{\tau}_{1}+\left[\hat{\boldsymbol{\eta}}_{2}^{\prime} \overline{\boldsymbol{\tau}}_{2}\right]^{\top} \overline{\boldsymbol{\tau}}_{1}\right)\right] \int \mathcal{D}\left(\boldsymbol{\eta}_{1}, \hat{\boldsymbol{\eta}}_{1}\right) \mathcal{D}\left(\boldsymbol{\eta}_{1}^{\prime}, \hat{\boldsymbol{\eta}}_{1}^{\prime}\right) \kappa_{p}\left(\boldsymbol{v}_{1}\right) \\
&= \prod_{\ell=2}^{L+1} \int \mathcal{D} \boldsymbol{x}_{\ell} \mathcal{D} \boldsymbol{x}_{\ell}^{\prime} \prod_{\mathbf{k}_{2}}\left\{\sum_{\tau, \bar{\tau}= \pm 1} \exp \left(i \hat{\eta}_{\mathbf{k}_{2}} \tau_{\mathbf{k}_{2}} \tau+i \hat{\eta}_{\mathbf{k}_{2}}^{\prime} \bar{\tau}_{\mathbf{k}_{2}} \bar{\tau}\right)\right. \\
&\left.\int_{0}^{\infty} \frac{\mathrm{d} \eta}{\sqrt{2 \pi}} \exp \left[-\frac{1}{2}\left(\eta-\tau X_{\left[\mathbf{k}_{2}\right]}\right)^{2}\right] \mathcal{H}\left(\bar{\tau}\left(\beta(p) \tau \eta+X_{\left[\mathbf{k}_{2}\right]}^{\prime}\right)\right)\right\}^{K_{1}}
\end{aligned}
$$

and by using the identities

$$
1=\int_{0}^{\infty} \frac{\mathrm{d} \eta}{\sqrt{2 \pi}}\left\{\exp \left[-\frac{1}{2}\left(\eta-X_{\left[\mathbf{k}_{2}\right]}\right)^{2}\right]+\exp \left[-\frac{1}{2}\left(\eta+X_{\left[\mathbf{k}_{2}\right]}\right)^{2}\right]\right\}
$$


$\operatorname{erf}\left(\frac{X_{\left[\mathbf{k}_{2}\right]}}{\sqrt{2}}\right)=\int_{0}^{\infty} \frac{\mathrm{d} \eta}{\sqrt{2 \pi}}\left\{\exp \left[-\frac{1}{2}\left(\eta-X_{\left[\mathbf{k}_{2}\right]}\right)^{2}\right]-\exp \left[-\frac{1}{2}\left(\eta+X_{\left[\mathbf{k}_{2}\right]}\right)^{2}\right]\right\}$

and the definitions

$\mathcal{G}_{p}^{+}\left(X_{\left[\mathbf{k}_{2}\right]}, X_{\left[\mathbf{k}_{2}\right]}^{\prime}\right)=\int \mathcal{D} \eta \operatorname{sgn}\left(\eta+X_{\left[\mathbf{k}_{2}\right]}\right) \operatorname{erf}\left(\frac{\beta(p)\left(\eta+X_{\left[\mathbf{k}_{2}\right]}\right)+X_{\left[\mathbf{k}_{2}\right]}^{\prime}}{\sqrt{2}}\right)$

$\mathcal{G}_{p}^{-}\left(X_{\left[\mathbf{k}_{2}\right]}, X_{\left[\mathbf{k}_{2}\right]}^{\prime}\right)=\int \mathcal{D} \eta \operatorname{erf}\left(\frac{\beta(p)\left(\eta+X_{\left[\mathbf{k}_{2}\right]}\right)+X_{\left[\mathbf{k}_{2}\right]}^{\prime}}{\sqrt{2}}\right)$,

we can define the function $\Lambda_{p}\left(\boldsymbol{v} ; X, X^{\prime}\right)$, where $\boldsymbol{v}=\left\{\hat{\eta}, \tau, \hat{\eta}^{\prime}, \bar{\tau}\right\}$

$$
\begin{aligned}
& \Lambda_{p}\left(\boldsymbol{v} ; X, X^{\prime}\right) \equiv R_{p}\left(\boldsymbol{v} ; X, X^{\prime}\right)+i I_{p}\left(\boldsymbol{v} ; X, X^{\prime}\right) \\
& R_{p}\left(\boldsymbol{v} ; X, X^{\prime}\right) \equiv \cos (\hat{\eta}) \cos \left(\hat{\eta}^{\prime}\right)-\tau \sin (\hat{\eta}) \bar{\tau} \sin \left(\hat{\eta}^{\prime}\right) \mathcal{G}_{p}^{+}\left(X, X^{\prime}\right) \\
& I_{p}\left(\boldsymbol{v} ; X, X^{\prime}\right) \equiv \tau \sin (\hat{\eta}) \cos \left(\hat{\eta}^{\prime}\right) \operatorname{erf}\left(\frac{X}{\sqrt{2}}\right)+\cos (\hat{\eta}) \bar{\tau} \sin \left(\hat{\eta}^{\prime}\right) \mathcal{G}_{p}^{-}\left(X, X^{\prime}\right) .
\end{aligned}
$$

With this definition we have that

$$
\kappa_{p}\left(\boldsymbol{v}_{2}\right)=\prod_{\ell=2}^{L+1} \int \mathcal{D} \boldsymbol{x}_{\ell} \mathcal{D} \boldsymbol{x}_{\ell}^{\prime} \prod_{\mathbf{k}_{2}}\left[\Lambda_{p}\left(\boldsymbol{v}_{\mathbf{k}_{2}} ; X_{\left[\mathbf{k}_{2}\right]}, X_{\left[\mathbf{k}_{2}\right]}^{\prime}\right)\right]^{K_{1}},
$$

where $\boldsymbol{v}_{\mathbf{k}_{2}}$ represents the set of variables $\hat{\eta}_{\mathbf{k}_{2}}, \hat{\eta}_{\mathbf{k}_{2}}^{\prime}, \tau_{\mathbf{k}_{2}}, \bar{\tau}_{\mathbf{k}_{2}}$.

By using the kernel of expression (A.7) we can reconstruct the average discrepancy component

$$
\begin{aligned}
\mathfrak{d}(p, \mathbf{Q})= & 2 \int \mathcal{D}\left(\eta_{L+1}, \hat{\eta}_{L+1}\right) \mathcal{D}\left(\eta_{L+1}^{\prime}, \hat{\eta}_{L+1}^{\prime}\right) \int \mathcal{D} x_{L+1} \mathcal{D} x_{L+1}^{\prime} \\
& \sum_{\left\{\boldsymbol{\tau}_{L}, \overline{\boldsymbol{\tau}}_{L}\right\}} \exp \left[i \sum_{\mathbf{k}_{L}}\left(\hat{\eta}_{L+1} \tau_{\mathbf{k}_{\ell}}-\hat{\eta}_{L+1}^{\prime} \bar{\tau}_{\mathbf{k}_{\ell}}\right)\right] \int \mathcal{D}\left(\boldsymbol{\eta}_{L}, \hat{\boldsymbol{\eta}}_{L}\right) \mathcal{D}\left(\boldsymbol{\eta}_{L}^{\prime}, \hat{\boldsymbol{\eta}}_{L}^{\prime}\right) \int \mathcal{D} \boldsymbol{x}_{L} \mathcal{D} \boldsymbol{x}_{L}^{\prime} \\
& \prod_{\ell=L-1}^{2} \sum_{\left\{\boldsymbol{\tau}_{\ell}, \overline{\boldsymbol{\tau}}_{\ell}\right\}} \exp \left[i\left(\left[\hat{\boldsymbol{\eta}}_{\ell+1} \boldsymbol{\tau}_{\ell+1}\right]^{\top} \boldsymbol{\tau}_{\ell}+\left[\hat{\boldsymbol{\eta}}_{\ell+1}^{\prime} \overline{\boldsymbol{\tau}}_{\ell+1}\right]^{\top} \overline{\boldsymbol{\tau}}_{\ell}\right)\right] \int \mathcal{D}\left(\boldsymbol{\eta}_{\ell}, \hat{\boldsymbol{\eta}}_{\ell}\right) \mathcal{D}\left(\boldsymbol{\eta}_{\ell}^{\prime}, \hat{\boldsymbol{\eta}}_{\ell}^{\prime}\right) \\
& \int \mathcal{D} \boldsymbol{x}_{\ell} \mathcal{D} \boldsymbol{x}_{\ell}^{\prime} \prod_{\mathbf{k}_{2}}\left[\Lambda_{p}\left(\boldsymbol{v}_{\mathbf{k}_{2}} ; X_{\left[\mathbf{k}_{2}\right]}, X_{\left[\mathbf{k}_{2}\right]}^{\prime}\right)\right]^{K_{1}}
\end{aligned}
$$

Observe that the function $\Lambda_{p}\left(\boldsymbol{v}_{\mathbf{k}_{2}} ; X_{\left[\mathbf{k}_{2}\right]}, X_{\left[\mathbf{k}_{2}\right]}^{\prime}\right)$ depends only on the variables described by the path $\mathbf{k}_{2}$ and does not depend on any interaction linking variables with different indexes.

In order to solve the problem analytically in the large number of hidden units regime, let us define the operators $\mathcal{U}_{\ell}$ for all $\ell=2,3, \ldots, L+1$

$$
\begin{aligned}
\boldsymbol{U}_{\ell} & \equiv \sum_{\left\{\tau_{\ell}, \bar{\tau}_{\ell}\right\}} \exp \left(i \hat{\eta}_{\ell+1} \tau_{\ell+1} \tau_{\ell}+i \hat{\eta}_{\ell+1}^{\prime} \bar{\tau}_{\ell+1} \bar{\tau}_{\ell}\right) \int \mathcal{D} x_{\ell} \mathcal{D} x_{\ell}^{\prime} \lim _{K_{\ell-1} \uparrow \infty} \int \mathcal{D}\left(\eta_{\ell}, \hat{\eta}_{\ell}\right) \mathcal{D}\left(\eta_{\ell}^{\prime}, \hat{\eta}_{\ell}^{\prime}\right) \\
\boldsymbol{U}_{L+1} & \equiv \sum_{\left\{\tau_{L+1}, \bar{\tau}_{L+1}\right\}} \delta_{\tau_{L+1}, 1} \delta_{\bar{\tau}_{L+1},-1} \int \mathcal{D} x_{L+1} \mathcal{D} x_{L+1}^{\prime} \lim _{K_{L} \uparrow \infty} \int \mathcal{D}\left(\eta_{L+1}, \hat{\eta}_{L+1}\right) \mathcal{D}\left(\eta_{L+1}^{\prime}, \hat{\eta}_{L+1}^{\prime}\right)
\end{aligned}
$$

With these operators we can write

$$
\mathfrak{d}_{p}(\mathbf{Q})=2 \mathcal{U}_{L+1}\left[\Lambda_{p}\left(\boldsymbol{v}_{L+1} ; X_{L+1}, X_{L+1}^{\prime}\right)\right]^{K_{L}}
$$


where

$$
\Lambda_{p}\left(\boldsymbol{v}_{\ell+1} ; X_{\ell+1}, X_{\ell+1}^{\prime}\right) \equiv \mathcal{U}_{\ell}\left[\Lambda_{p}\left(\boldsymbol{v}_{\ell} ; X_{\ell}, X_{\ell}^{\prime}\right)\right]^{K_{\ell-1}}
$$

where $\boldsymbol{v}_{\ell}$ represent the set of variables $\hat{\eta}_{\ell}, \hat{\eta}_{\ell}^{\prime}, \tau_{\ell}, \bar{\tau}_{\ell}$ and $X_{\ell} \equiv \sum_{j=\ell}^{L+1} \frac{\phi_{j}}{\phi_{1}} x_{j}$ (and equivalently for $X_{\ell}^{\prime}$ ).

The set of equations (A.8) with the iteration of order 1 given by (A.6) can be analytically solved if the number of hidden units at each layer is large (i.e., in the limit of $K_{\ell} \rightarrow \infty$ for all $\left.\ell=1,2, \ldots, L\right)$. First of all let us define the variables

$$
\tilde{X}_{\ell} \equiv\left(\zeta_{\ell-1}-\frac{\zeta_{\ell}}{K_{\ell}}\right)^{\frac{1}{2}} x_{\ell}+K_{\ell}^{-\frac{1}{2}} \tilde{X}_{\ell+1}
$$

with $\tilde{X}_{L+1} \equiv \zeta_{L}^{\frac{1}{2}} x_{L+1}$ such that

$$
\begin{aligned}
X_{\ell} & =\sum_{j=\ell}^{L+1} \frac{\phi_{j}}{\phi_{1}} x_{j} \\
& =\left(1-\frac{\zeta_{1}}{K_{1}}\right)^{-\frac{1}{2}} K_{\ell-1}^{-\frac{1}{2}} \tilde{X}_{\ell} \\
& \simeq K_{\ell-1}^{-1 / 2}\left[\left(\zeta_{\ell-1}-\frac{\zeta_{\ell}}{K_{\ell}}\right)^{\frac{1}{2}} x_{\ell}+K_{\ell}^{-\frac{1}{2}} \tilde{X}_{\ell+1}\right]+O\left(\left(K_{1} K_{\ell-1}\right)^{-\frac{1}{2}}\right),
\end{aligned}
$$

and

$$
\tilde{Y}_{\ell} \equiv \frac{\beta \tilde{X}_{\ell}+\tilde{X}_{\ell}^{\prime}}{\sqrt{1+\beta^{2}}} .
$$

Using the fact that the number of hidden units is large we can expand (A.4), (A.5) and the error function, therefore

$$
\begin{aligned}
& \mathcal{G}_{p}^{+}\left(X_{2}, X_{2}^{\prime}\right) \simeq \mathcal{G}_{1}(p)+O\left(K_{1}^{-1}\right) \\
& \mathcal{G}_{p}^{-}\left(X_{2}, X_{2}^{\prime}\right) \simeq \operatorname{erf}\left(\alpha_{1} \tilde{Y}_{2} K_{1}^{-\frac{1}{2}}\right)+O\left(K_{1}^{-\frac{3}{2}}\right) \\
& \operatorname{erf}\left(\frac{X_{2}}{\sqrt{2}}\right)=\operatorname{erf}\left(\alpha_{1} \tilde{X}_{2} K_{1}^{-\frac{1}{2}}\right),
\end{aligned}
$$

where $\mathcal{G}_{1}(p) \equiv 1-\frac{2}{\pi} \arccos (1-2 p)$ and $\alpha_{1}=2^{-\frac{1}{2}}$. Before proceeding with the calculation let us express the real and imaginary parts of the function $\Lambda_{p}$ using these approximations, and disregarding terms of $O\left(K_{1}^{-1}\right)$

$R_{p}\left(\boldsymbol{v}_{2} ; X_{2}, X_{2}^{\prime}\right) \simeq \cos \left(\hat{\eta}_{2}\right) \cos \left(\hat{\eta}_{2}^{\prime}\right)-\tau_{2} \bar{\tau}_{2} \sin \left(\hat{\eta}_{2}\right) \sin \left(\hat{\eta}_{2}^{\prime}\right) \mathcal{G}_{1}(p)$

$I_{p}\left(\boldsymbol{v}_{2} ; X_{2}, X_{2}^{\prime}\right) \simeq \tau_{2} \sin \left(\hat{\eta}_{2}\right) \cos \left(\hat{\eta}_{2}^{\prime}\right) \operatorname{erf}\left(\alpha_{1} \tilde{X}_{2} K_{1}^{-\frac{1}{2}}\right)+\bar{\tau}_{2} \cos \left(\hat{\eta}_{2}\right) \sin \left(\hat{\eta}_{2}^{\prime}\right) \operatorname{erf}\left(\alpha_{1} \tilde{Y}_{2} K_{1}^{-\frac{1}{2}}\right)$

therefore

$$
\begin{gathered}
\Lambda_{p}^{K_{1}}\left(\boldsymbol{v}_{2} ; X_{2}, X_{2}^{\prime}\right)=\left[R_{p}\left(\boldsymbol{v}_{2} ; X_{2}, X_{2}^{\prime}\right)+i I_{p}\left(\boldsymbol{v}_{2} ; X_{2}, X_{2}^{\prime}\right)\right]^{K_{1}} \\
=\sum_{k=0}^{K_{1}} \sum_{r=0}^{K_{1}-k} \sum_{j=0}^{k} i^{k}\left(\begin{array}{c}
K_{1} \\
k
\end{array}\right)\left(\begin{array}{c}
K_{1}-k \\
r
\end{array}\right)\left(\begin{array}{c}
k \\
j
\end{array}\right) \tau_{2}^{r+k-j} \bar{\tau}_{2}^{r+j}\left(-\mathcal{G}_{p}^{+}\left(X_{2}, X_{2}^{\prime}\right)\right)^{r}\left(\mathcal{G}_{p}^{-}\left(X_{2}, X_{2}^{\prime}\right)\right)^{j} \\
\quad \operatorname{erf}\left(\alpha_{1} \tilde{X}_{2} K_{1}^{-\frac{1}{2}}\right)^{2 m+1} \cos \left(\hat{\eta}_{2}\right)^{K_{1}-k-r+j} \sin \left(\hat{\eta}_{2}\right)^{k+r-j} \cos \left(\hat{\eta}_{2}^{\prime}\right)^{K_{1}-r-j} \sin \left(\hat{\eta}_{2}^{\prime}\right)^{r+j},
\end{gathered}
$$


thus

$$
\begin{aligned}
\int \mathcal{D} & \left(\eta_{2}, \hat{\eta}_{2}\right) \mathcal{D}\left(\eta_{2}^{\prime}, \hat{\eta}_{2}^{\prime}\right) \Lambda_{p}^{K_{1}}\left(\boldsymbol{v}_{2} ; X_{2}, X_{2}^{\prime}\right)=\sum_{k=0}^{K_{1}} \sum_{r=0}^{K_{1}-k} \sum_{j=0}^{k} i^{k}\left(\begin{array}{c}
K_{1} \\
k
\end{array}\right)\left(\begin{array}{c}
K_{1}-k \\
r
\end{array}\right)\left(\begin{array}{c}
k \\
j
\end{array}\right) \tau_{2}^{r+k-j} \bar{\tau}_{2}^{r+j} \\
& \times\left(-\mathcal{G}_{p}^{+}\left(X_{2}, X_{2}^{\prime}\right)\right)^{r}\left(\mathcal{G}_{p}^{-}\left(X_{2}, X_{2}^{\prime}\right)\right)^{j} \operatorname{erf}\left(\alpha_{1} \tilde{X}_{2} K_{1}^{-\frac{1}{2}}\right)^{k-j} \\
& \times \int \mathcal{D}\left(\eta_{2}, \hat{\eta}_{2}\right) \cos \left(\hat{\eta}_{2}\right)^{K_{1}-k-r+j} \sin \left(\hat{\eta}_{2}\right)^{k+r-j} \int \mathcal{D}\left(\eta_{2}^{\prime}, \hat{\eta}_{2}^{\prime}\right) \cos \left(\hat{\eta}_{2}^{\prime}\right)^{K_{1}-r-j} \sin \left(\hat{\eta}_{2}^{\prime}\right)^{r+j}(\mathrm{~A} .12)
\end{aligned}
$$

and in agreement with (C.4), (C.5) and (C.6) we have that there are four situations where the integrals in (A.12) are not zero

(i) $k+r-j=r+j=0$, which implies that $k=r=j=0$. The correspondent term to the series evaluates to $\frac{1}{4}$.

(ii) $k+r-j=0$ and $r+j \neq 0$ is odd, which implies that $r=0$, given that $j \leq k$. Thus $k=j$ is odd.

(iii) $k+r-j \neq 0$ is odd, and $r+j=0$ which implies that $r=j=0$, given that $0 \leq r, j$. Thus $k$ is odd.

(iv) $k+r-j \neq 0$ is odd,and $r+j \neq 0$ is odd, which implies that $k$ is even and $r$ and $j$ have different parities.

The first term, with all the indexes equal to 0 is straightforwardly $A_{0}=\frac{1}{4}$. The second term has the indexes $j=k=2 m+1$ and $r=0$. Thus, using that $K_{1}=2 M+1$

$$
\begin{aligned}
A_{1}^{M}\left(\bar{\tau}_{2}, \tilde{Y}_{2}\right) & =\sum_{m=0}^{M}\left(\begin{array}{c}
2 M+1 \\
2 m+1
\end{array}\right)\left(i \bar{\tau}_{2}\right)^{2 m+1}\left(\mathcal{G}_{p}^{-}\left(X_{2}, X_{2}^{\prime}\right)\right)^{2 m+1} \frac{1}{2} a_{m}^{M} \\
& =\frac{\bar{\tau}_{2}}{2^{2 M+2}} \sum_{m=0}^{M}(-1)^{m}\left(\begin{array}{c}
2 M+1 \\
2 m+1
\end{array}\right)\left(\begin{array}{c}
M \\
m
\end{array}\right)^{-1}\left(\begin{array}{c}
2 m \\
m
\end{array}\right)\left(\begin{array}{c}
2(M-m) \\
M-m
\end{array}\right)\left(\mathcal{G}_{p}^{-}\left(X_{2}, X_{2}^{\prime}\right)\right)^{2 m+1},
\end{aligned}
$$

where $a_{m}^{M}$ is given by (C.6)

$$
a_{m}^{M}=-\frac{i}{2^{2 M+1}}\left(\begin{array}{c}
M \\
m
\end{array}\right)^{-1}\left(\begin{array}{c}
2 m \\
m
\end{array}\right)\left(\begin{array}{c}
2(M-m) \\
M-m
\end{array}\right) .
$$

By using (A.10) we can take the limit of $K_{1} \rightarrow \infty$

$$
\begin{aligned}
A_{1}\left(\bar{\tau}_{2}, \tilde{Y}_{2}\right) & =\frac{\bar{\tau}_{2}}{4} \lim _{M \uparrow \infty} \sum_{m=0}^{M}(-1)^{m} \frac{\left(\begin{array}{c}
2 M+1 \\
2 m+1
\end{array}\right)\left(\begin{array}{c}
2 m \\
m
\end{array}\right)\left(\begin{array}{c}
2(M-m) \\
M-m
\end{array}\right)}{2^{2 M}\left(\begin{array}{c}
M \\
m
\end{array}\right)(2 M+1)^{m+\frac{1}{2}}}\left(\frac{2}{\sqrt{\pi}} \alpha_{1} \tilde{Y}_{2}\right)^{2 m+1} \\
& =\frac{\bar{\tau}_{2}}{4} \lim _{M \uparrow \infty} \frac{2}{\sqrt{\pi}} \sum_{m=0}^{M}\left[\frac{(-1)^{m}}{(2 m+1) m !}\left(\sqrt{\frac{2}{\pi}} \alpha_{1} \tilde{Y}_{2}\right)^{2 m+1}+O\left(M^{-1}\right)\right] \\
& =\frac{\bar{\tau}_{2}}{4} \operatorname{erf}\left(\sqrt{\frac{2}{\pi}} \alpha_{1} \tilde{Y}_{2}\right) .
\end{aligned}
$$

Therefore, the integral over the Hubbard-Stratonovitch variables $x_{2}$ and $x_{2}^{\prime}$ is, using the recursive nature of $\tilde{X}_{2}$ and $\tilde{X}_{2}^{\prime}$. Therefore

$$
\int \mathcal{D} x_{2} \mathcal{D} x_{2}^{\prime} A_{1}\left(\bar{\tau}_{2}, \tilde{Y}_{2}\right)=\frac{\bar{\tau}_{2}}{4} \mathscr{A}_{1}\left(\tilde{Y}_{3}\right)
$$


such that

$$
\begin{aligned}
& \mathscr{A}_{1}\left(\tilde{Y}_{3}\right) \equiv \int \mathcal{D} x_{2} \mathcal{D} x_{2}^{\prime} \operatorname{erf}\left(\sqrt{\frac{2}{\pi}} \alpha_{1} \frac{\beta \tilde{X}_{2}+\tilde{X}_{2}^{\prime}}{\sqrt{1+\beta^{2}}}\right) \\
& \simeq \int \mathcal{D} x_{2} \mathcal{D} x_{2}^{\prime}\left\{\operatorname{erf}\left(\sqrt{\frac{2 \zeta_{1}}{\pi}} \alpha_{1} \frac{\beta x_{2}+x_{2}^{\prime}}{\sqrt{1+\beta^{2}}}\right)+\frac{2^{\frac{3}{2}} \alpha_{1}}{\pi} \tilde{Y}_{3} K_{2}^{-\frac{1}{2}} \exp \left[-\frac{2 \zeta_{1} \alpha_{1}^{2}}{\pi} \frac{\left(\beta x_{2}+x_{2}^{\prime}\right)^{2}}{1+\beta^{2}}\right]\right\} \\
& \quad+O\left(K_{2}^{-\frac{3}{2}}\right) \\
& \simeq \frac{2}{\sqrt{\pi}} \sqrt{\frac{2 \alpha_{1}^{2}}{\pi+4 \zeta_{1} \alpha_{1}^{2}}} \tilde{Y}_{3} K_{2}^{-\frac{1}{2}}+O\left(K_{2}^{-\frac{3}{2}}\right) \\
& \simeq \operatorname{erf}\left(\alpha_{2} \tilde{Y}_{3} K_{2}^{-\frac{1}{2}}\right)+O\left(K_{2}^{-\frac{3}{2}}\right),
\end{aligned}
$$

where

$$
\alpha_{2} \equiv \sqrt{\frac{2 \alpha_{1}^{2}}{\pi+4 \zeta_{1} \alpha_{1}^{2}}} .
$$

The third term is similar to the second. It is formed by the following contributions

$$
A_{2}^{M}\left(\tau_{2}, \tilde{X}_{2}\right)=\sum_{m=0}^{M}\left(\begin{array}{c}
2 M+1 \\
2 m+1
\end{array}\right)\left(i \tau_{2}\right)^{2 m+1} \operatorname{erf}\left(\alpha_{1} \tilde{X}_{2} K_{1}^{-\frac{1}{2}}\right)^{2 m+1} \frac{1}{2} a_{m}^{M},
$$

and by expanding the error function, taken the limit of large $M$ and integrating over $x_{2}$ and $x_{2}^{\prime}$, we arrive to

$$
\begin{aligned}
\int \mathcal{D} x_{2} \mathcal{D} x_{2}^{\prime} A_{2}\left(\tau_{2}, \tilde{X}_{2}\right) & =\frac{\tau_{2}}{4} \mathscr{A}_{2}\left(\tilde{X}_{3}\right), \\
\mathscr{A}_{2}\left(\tilde{X}_{3}\right) & \simeq \operatorname{erf}\left(\alpha_{2} \tilde{X}_{3} K_{2}^{-\frac{1}{2}}\right)+O\left(K_{2}^{-\frac{3}{2}}\right) .
\end{aligned}
$$

The last contribution involves terms with even values of $k$ and non-zero, uneven values of $r$ and $j$. Therefore

$$
\begin{gathered}
A_{3}^{M}\left(\tau_{2}, \bar{\tau}_{2}, \tilde{X}_{2}, \tilde{Y}_{2}\right)=\tau_{2} \bar{\tau}_{2} \sum_{m=0}^{M} \sum_{n=0}^{M-m} \sum_{s=0}^{m-1}(-1)^{m}\left(\begin{array}{c}
2 M+1 \\
2 m
\end{array}\right)\left(\begin{array}{c}
2(M-m)+1 \\
2 n
\end{array}\right)\left(\begin{array}{c}
2 m \\
2 s+1
\end{array}\right) \\
a_{n+s}^{M} a_{m+n-s-1}^{M}\left(\mathcal{G}_{p}^{+}\left(X_{2}, X_{2}^{\prime}\right)\right)^{2 n} \operatorname{erf}\left(\alpha_{1} \tilde{X}_{2} K_{1}^{-\frac{1}{2}}\right)^{2 m-2 s-1}\left(\mathcal{G}_{p}^{-}\left(X_{2}, X_{2}^{\prime}\right)\right)^{2 s+1}- \\
-\tau_{2} \bar{\tau}_{2} \sum_{m=0}^{M} \sum_{n=0}^{M-m} \sum_{s=0}^{m}(-1)^{m}\left(\begin{array}{c}
2 M+1 \\
2 m
\end{array}\right)\left(\begin{array}{c}
2(M-m)+1 \\
2 n+1
\end{array}\right)\left(\begin{array}{c}
2 m \\
2 s
\end{array}\right) \\
a_{n+s}^{M} a_{m+n-s}^{M}\left(\mathcal{G}_{p}^{+}\left(X_{2}, X_{2}^{\prime}\right)\right)^{2 n+1} \operatorname{erf}\left(\alpha_{1} \tilde{X}_{2} K_{1}^{-\frac{1}{2}}\right)^{2 m-2 s}\left(\mathcal{G}_{p}^{-}\left(X_{2}, X_{2}^{\prime}\right)\right)^{2 s} .
\end{gathered}
$$

In order to continue the development we introduce the following identity

$$
\int_{-\infty}^{\infty} \mathcal{D} y y^{2 \ell}=\left\{\begin{array}{lr}
1 & \ell=0 \\
(2 \ell-1) ! ! & \text { otherwise }
\end{array}\right.
$$

Therefore, for large values of $M$ and using the expansions (A.10) and (A.11) we have that the leading terms in $M$ are

$$
\left(\begin{array}{c}
2 M+1 \\
2 m
\end{array}\right)\left(\begin{array}{c}
2(M-m)+1 \\
2 n
\end{array}\right) a_{n+s}^{M} a_{m+n-s-1}^{M} \operatorname{erf}\left(\alpha_{1} \tilde{X}_{2} K_{1}^{-\frac{1}{2}}\right)^{2 m-2 s-1}\left(\mathcal{G}_{p}^{-}\left(X_{2}, X_{2}^{\prime}\right)\right)^{2 s+1}
$$


$\simeq-\frac{1}{2 \pi} \frac{1}{(2 m) !(2 n) !}\left(\frac{2}{\sqrt{\pi}} \alpha_{1} \tilde{X}_{2}\right)^{2 m-2 s-1}\left(\frac{2}{\sqrt{\pi}} \alpha_{1} \tilde{Y}_{2}\right)^{2 s+1} \int \mathcal{D} y \mathcal{D} z y^{2(m+n-s-1)} z^{2(n+s)}$

and

$$
\begin{aligned}
& \left(\begin{array}{c}
2 M+1 \\
2 m
\end{array}\right)\left(\begin{array}{c}
2(M-m)+1 \\
2 n+1
\end{array}\right) a_{n+s}^{M} a_{m+n-s}^{M} \operatorname{erf}\left(\alpha_{2} \tilde{X}_{2} K_{1}^{-\frac{1}{2}}\right)^{2 m-2 s}\left(\mathcal{G}_{p}^{-}\left(X_{2}, X_{2}^{\prime}\right)\right)^{2 s} \\
& \simeq-\frac{1}{2 \pi} \frac{1}{(2 m) !(2 n+1) !}\left(\frac{2}{\sqrt{\pi}} \alpha_{1} \tilde{X}_{2}\right)^{2 m-2 s}\left(\frac{2}{\sqrt{\pi}} \alpha_{1} \tilde{Y}_{2}\right)^{2 s} \int \mathcal{D} y \mathcal{D} z y^{2(m+n-s)} z^{2(n+s)}
\end{aligned}
$$

so we can reconstruct the expression for $A_{3}^{M}$ such that

$$
\begin{aligned}
A_{3}^{M}\left(\tau_{2}, \bar{\tau}_{2}, \tilde{X}_{2}, \tilde{Y}_{2}\right) \simeq & -\frac{\tau_{2} \bar{\tau}_{2}}{2 \pi} \int \frac{\mathcal{D} y}{y} \frac{\mathcal{D} z}{z} \sum_{m=0}^{M} \frac{(-1)^{m}}{(2 m) !} \sum_{n=0}^{M-m} \frac{\left(y z \mathcal{G}_{1}(p)\right)^{2 n}}{(2 n) !} \\
& \times \sum_{s=0}^{m-1}\left(\begin{array}{c}
2 m \\
2 s+1
\end{array}\right)\left(\frac{2}{\sqrt{\pi}} y \alpha_{1} \tilde{X}_{2}\right)^{2 m-2 s-1}\left(\frac{2}{\sqrt{\pi}} z \alpha_{1} \tilde{Y}_{2}\right)^{2 s+1} \\
& +\frac{\tau_{2} \bar{\tau}_{2}}{2 \pi} \int \frac{\mathcal{D} y}{y} \frac{\mathcal{D} z}{z} \sum_{m=0}^{M} \frac{(-1)^{m}}{(2 m) !} \sum_{n=0}^{M-m} \frac{\left(y z \mathcal{G}_{1}(p)\right)^{2 n+1}}{(2 n+1) !} \\
& \times \sum_{s=0}^{m}\left(\begin{array}{c}
2 m \\
2 s
\end{array}\right)\left(\frac{2}{\sqrt{\pi}} y \alpha_{1} \tilde{X}_{2}\right)^{2 m-2 s}\left(\frac{2}{\sqrt{\pi}} z \alpha_{1} \tilde{Y}_{2}\right)^{2 s}+O\left(K_{1}^{-1}\right) .
\end{aligned}
$$

If $M\left(K_{1}\right)$ is large enough, the sums in $n$ can be approximated by the hyperbolic cosine and sine, respectively. Thus, in the limit,

$$
\begin{aligned}
& A_{3}\left(\tau_{2}, \bar{\tau}_{2}, \tilde{X}_{2}, \tilde{Y}_{2}\right)=\frac{\tau_{2} \bar{\tau}_{2}}{2 \pi} \int \frac{\mathcal{D} y}{y} \frac{\mathcal{D} z}{z} \sum_{m=0}^{\infty} \frac{(-1)^{m}}{(2 m) !} \\
& \times\left[-\cosh \left(y z \mathcal{G}_{1}(p)\right) \sum_{s=0}^{m-1}\left(\begin{array}{c}
2 m \\
2 s+1
\end{array}\right)\left(\frac{2}{\sqrt{\pi}} y \alpha_{1} \tilde{X}_{2}\right)^{2 m-2 s-1}\left(\frac{2}{\sqrt{\pi}} z \alpha_{1} \tilde{Y}_{2}\right)^{2 s+1}\right. \\
& \left.+\sinh \left(y z \mathcal{G}_{1}(p)\right) \sum_{s=0}^{m}\left(\begin{array}{c}
2 m \\
2 s
\end{array}\right)\left(\frac{2}{\sqrt{\pi}} y \alpha_{1} \tilde{X}_{2}\right)^{2 m-2 s}\left(\frac{2}{\sqrt{\pi}} z \alpha_{1} \tilde{Y}_{2}\right)^{2 s}\right]
\end{aligned}
$$

which can be rearranged such that

$$
\begin{aligned}
& A_{3}\left(\tau_{2}, \bar{\tau}_{2}, \tilde{X}_{2}, \tilde{Y}_{2}\right)=\frac{\tau_{2} \bar{\tau}_{2}}{4 \pi} \int \frac{\mathcal{D} y}{y} \frac{\mathcal{D} z}{z} \\
& \quad\left[\exp \left(y z \mathcal{G}_{1}(p)\right) \cos \left(\frac{2 \alpha_{1}}{\sqrt{\pi}}\left(y \tilde{X}_{2}-z \tilde{Y}_{2}\right)\right)-\exp \left(-y z \mathcal{G}_{1}(p)\right) \cos \left(\frac{2 \alpha_{1}}{\sqrt{\pi}}\left(y \tilde{X}_{2}+z \tilde{Y}_{2}\right)\right)\right](A
\end{aligned}
$$

The next step involves the integration of the Hubbard-Stratonovitch variables. Observe that only even functions in such variables will survive the process. $A_{3}$ can be expressed as a linear combination of an odd and an even functions in $x_{2}$ and $x_{2}^{\prime}$. The even part is, disregarding corrections of $O\left(K_{2}^{-1}\right)$,

$$
\begin{aligned}
A_{3}^{\text {even }}\left(\tau_{2}, \bar{\tau}_{2}, \tilde{X}_{2}, \tilde{Y}_{2}\right) \simeq \frac{\tau_{2} \bar{\tau}_{2}}{4 \pi} \int \frac{\mathcal{D} y}{y} \frac{\mathcal{D} z}{z} \\
\left\{\exp \left(y z \mathcal{G}_{1}(p)\right) \cos \left[\sqrt{\frac{4 \zeta_{1} \alpha_{1}^{2}}{\pi}}\left(y-\frac{\beta z}{\sqrt{1+\beta^{2}}}\right) x_{2}\right] \cos \left(\sqrt{\frac{4 \zeta_{1} \alpha_{1}^{2}}{\pi}} \frac{z}{\sqrt{1+\beta^{2}}} x_{2}^{\prime}\right)\right.
\end{aligned}
$$


$\left.-\exp \left(-y z \mathcal{G}_{1}(p)\right) \cos \left[\sqrt{\frac{4 \zeta_{1} \alpha_{1}^{2}}{\pi}}\left(y+\frac{\beta z}{\sqrt{1+\beta^{2}}}\right) x_{2}\right] \cos \left(\sqrt{\frac{4 \zeta_{1} \alpha_{1}^{2}}{\pi}} \frac{z}{\sqrt{1+\beta^{2}}} x_{2}^{\prime}\right)\right\}$

which implies that

$$
\begin{aligned}
& \frac{\tau_{2} \bar{\tau}_{2}}{4} \mathscr{A}_{3}\left(\tilde{X}_{3}, \tilde{Y}_{3}\right) \equiv \int \mathcal{D} x_{2} \mathcal{D} x_{2}^{\prime} A_{3}\left(\tau_{2}, \bar{\tau}_{2}, \tilde{X}_{2}, \tilde{Y}_{2}\right) \\
& \mathscr{A}_{3}\left(\tilde{X}_{3}, \tilde{Y}_{3}\right) \simeq \frac{1}{\pi} \int \frac{\mathcal{D} y}{y} \frac{\mathcal{D} z}{z}\left\{\exp \left[y z \mathcal{G}_{1}(p)-\frac{2 \zeta_{1} \alpha_{1}^{2}}{\pi}\left(y-\frac{\beta z}{\sqrt{1+\beta^{2}}}\right)^{2}-\frac{2 \zeta_{1} \alpha_{1}^{2}}{\pi} \frac{z^{2}}{1+\beta^{2}}\right]-\right. \\
& \left.\quad-\exp \left[-y z \mathcal{G}_{1}(p)-\frac{2 \zeta_{1} \alpha_{1}^{2}}{\pi}\left(y+\frac{\beta z}{\sqrt{1+\beta^{2}}}\right)^{2}-\frac{2 \zeta_{1} \alpha_{1}^{2}}{\pi} \frac{z^{2}}{1+\beta^{2}}\right]\right\}+O\left(K_{2}^{-1}\right)
\end{aligned}
$$

and by defining the variables $u \equiv \sqrt{1+\frac{4}{\pi} \zeta_{1} \alpha_{1}^{2}} y$ and $t \equiv \sqrt{1+\frac{4}{\pi} \zeta_{1} \alpha_{1}^{2}} z$, and using that $1-2 p=\beta / \sqrt{1+\beta^{2}}$, we have that

$$
\mathscr{A}_{3}\left(\tilde{X}_{3}, \tilde{Y}_{3}\right) \simeq \frac{2}{\pi} \tau_{2} \bar{\tau}_{2} \int \frac{\mathcal{D} u}{u} \frac{\mathcal{D} t}{t} \sinh \left(\frac{\mathcal{G}_{2}(p)+\frac{4}{\pi} \zeta_{1} \alpha_{1}^{2}(1-2 p)}{1+\frac{4}{\pi} \zeta_{1} \alpha_{1}^{2}} u t\right)+O\left(K_{2}^{-1}\right) .
$$

This integral can be solved by expanding the hyperbolic sine in power series and using (A.13) backwards. Thus

$$
\begin{aligned}
\mathcal{J}(b) & \equiv \frac{2}{\pi} \int \frac{\mathcal{D} u}{u} \frac{\mathcal{D} t}{t} \sinh (b u t) \\
& =\frac{2}{\pi} \sum_{j=0}^{\infty} \frac{b^{2 j+1}}{(2 j+1) !}\left(\int \mathcal{D} u u^{2 j}\right)^{2} \\
& =\frac{2}{\pi}\left(b+\sum_{j=1}^{\infty} \frac{[(2 j-1) ! !]^{2}}{(2 j+1) !} b^{2 j+1}\right) \\
& =1-\frac{2}{\pi} \arccos (b) .
\end{aligned}
$$

In the end we obtain

$$
\begin{aligned}
\mathscr{A}_{3}\left(\tilde{X}_{3}, \tilde{Y}_{3}\right) & \simeq 1-\frac{2}{\pi} \arccos \left(\frac{\mathcal{G}_{1}(p)+\frac{4}{\pi} \zeta_{1} \alpha_{1}^{2}(1-2 p)}{1+\frac{4}{\pi} \zeta_{1} \alpha_{1}^{2}}\right)+O\left(K_{2}^{-1}\right) \\
& \simeq \mathcal{G}_{2}(p)+O\left(K_{2}^{-1}\right)
\end{aligned}
$$

where

$$
\begin{aligned}
\gamma_{1} & \equiv \frac{4 \zeta_{1} \alpha_{1}^{2}}{\pi+4 \zeta_{1} \alpha_{1}^{2}} \\
\mathcal{G}_{2}(p) & \equiv 1-\frac{2}{\pi} \arccos \left(\left(1-\gamma_{1}\right) \mathcal{G}_{1}(p)+\gamma_{1}(1-2 p)\right)
\end{aligned}
$$

The last operation we have to perform is the trace over the activation variables, i.e.,

$$
\begin{aligned}
& \Lambda_{p}\left(\boldsymbol{v}_{3} ; X_{3}, X_{3}^{\prime}\right)=\mathcal{U}_{2}\left[\Lambda_{p}\left(\boldsymbol{v}_{2} ; X_{2}, X_{2}^{\prime}\right)\right]^{K_{1}} \\
& =\frac{1}{4} \sum_{\left\{\tau_{2}, \bar{\tau}_{2}\right\}} \exp \left(i \hat{\eta}_{3} \tau_{3} \tau_{2}+i \hat{\eta}_{3}^{\prime} \bar{\tau}_{3} \bar{\tau}_{2}\right)\left(1+\bar{\tau}_{2} \operatorname{erf}\left(\alpha_{2} \tilde{Y}_{3} K_{2}^{-\frac{1}{2}}\right)+\tau_{2} \operatorname{erf}\left(\alpha_{2} \tilde{X}_{3} K_{2}^{-\frac{1}{2}}\right)+\tau_{2} \bar{\tau}_{2} \mathcal{G}_{2}(p)\right) \\
& =R_{p}\left(\boldsymbol{v}_{3} ; X_{3}, X_{3}^{\prime}\right)+i I_{p}\left(\boldsymbol{v}_{3} ; X_{3}, X_{3}^{\prime}\right)
\end{aligned}
$$


where the real and imaginary part of the function $\Lambda_{p}$ are, disregarding terms of $O\left(K_{2}^{-1}\right)$ and $O\left(K_{2}^{-\frac{3}{2}}\right)$ respectively

$R_{p}\left(\boldsymbol{v}_{3} ; X_{3}, X_{3}^{\prime}\right) \simeq \cos \left(\hat{\eta}_{3}\right) \cos \left(\hat{\eta}_{3}^{\prime}\right)-\tau_{3} \bar{\tau}_{3} \sin \left(\hat{\eta}_{3}\right) \sin \left(\hat{\eta}_{3}^{\prime}\right) \mathcal{G}_{2}(p)$

$I_{p}\left(\boldsymbol{v}_{3} ; X_{3}, X_{3}^{\prime}\right) \simeq \tau_{3} \sin \left(\hat{\eta}_{3}\right) \cos \left(\hat{\eta}_{3}^{\prime}\right) \operatorname{erf}\left(\alpha_{2} \tilde{X}_{3} K_{2}^{-\frac{1}{2}}\right)+\bar{\tau}_{3} \cos \left(\hat{\eta}_{3}\right) \sin \left(\hat{\eta}_{3}^{\prime}\right) \operatorname{erf}\left(\alpha_{2} \tilde{Y}_{3} K_{2}^{-\frac{1}{2}}\right)$.

The iteration the produces the functions of the variables at the $\ell+1$-th layer will have the following parameters

$$
\begin{aligned}
& \alpha_{\ell}^{2} \equiv \frac{2 \alpha_{\ell-1}^{2}}{\pi+4 \zeta_{\ell-1} \alpha_{\ell-1}^{2}}=\frac{1}{2}\left(\frac{2}{\pi}\right)^{\ell-1}\left[1+\sum_{j=1}^{\ell-1}\left(\frac{2}{\pi}\right)^{j} \zeta_{j}\right]^{-1} \\
& \gamma_{\ell} \equiv \frac{4 \zeta_{\ell} \alpha_{\ell}^{2}}{\pi+4 \zeta_{\ell} \alpha_{\ell}^{2}}=\left(\frac{2}{\pi}\right)^{\ell} \zeta_{\ell}\left[1+\sum_{j=1}^{\ell}\left(\frac{2}{\pi}\right)^{j} \zeta_{j}\right]^{-1} \\
& \mathcal{G}_{\ell+1}(p) \equiv 1-\frac{2}{\pi} \arccos \left(\left(1-\gamma_{\ell}\right) \mathcal{G}_{\ell}(p)+\gamma_{\ell}(1-2 p)\right)
\end{aligned}
$$

with the initial conditions

$$
\begin{aligned}
& \alpha_{1}^{2}=\frac{1}{2} \\
& \mathcal{G}_{1}(p)=1-\frac{2}{\pi} \arccos (1-2 p) .
\end{aligned}
$$

Observe that this process can be repeated until the very last iteration, where $\tilde{X}_{L+2}=$ $\tilde{Y}_{L+2}=0$. Therefore

$$
\mathfrak{d}(p, \mathbf{Q})=\frac{1}{\pi} \arccos \left(\left(1-\gamma_{L}\right) \mathcal{G}_{L}(p)+\gamma_{L}(1-2 p)\right) .
$$

\section{Appendix B. Calculation of the partial overlap matrix probability}

Let us suppose that we have selected $K$ vectors from $\mathbb{S}^{N}$ such there is a constraint on the angle between any pair of them, such that as a result we can construct the matrix Q. Let us find now the distribution probability of the matrix elements correspondent to the partial sums of the type $\frac{1}{N} \sum_{j \in \mathbb{I}_{P}} w_{\mathbf{k}_{1}, j} w_{\mathbf{k}_{1}^{\prime}, j}$. With this end we define the following quantities

$$
\begin{aligned}
r_{\mathbf{k}_{1}}^{2} & \equiv \frac{1}{N} \sum_{j \in \mathbb{I}_{P}} w_{\mathbf{k}_{1}, j}^{2} \\
r_{\mathbf{k}_{1}} r_{\mathbf{k}_{1}^{\prime}} \cos \left(\varphi_{\mathbf{k}_{1} \mathbf{k}_{1}^{\prime}}\right) & \equiv \frac{1}{N} \sum_{j \in \mathbb{I}_{P}} w_{\mathbf{k}_{1}, j} w_{\mathbf{k}_{1}^{\prime}, j}
\end{aligned}
$$

so the task is to find the distribution of the variables $r_{\mathbf{k}_{1}}^{2}$ (diagonal elements) and $r_{\mathbf{k}_{1}} r_{\mathbf{k}_{1}^{\prime}} \cos \left(\varphi_{\mathbf{k}_{1} \mathbf{k}_{1}^{\prime}}\right)$ (off-diagonal elements).

It is then

$$
\mathcal{P}\left(r_{\mathbf{k}_{1}}^{2}\right)=\int \mathrm{d} \mathbf{w}_{\mathbf{k}_{1}} \mathcal{P}\left(r_{\mathbf{k}_{1}}^{2} \mid \mathbf{w}_{\mathbf{k}_{1}}\right) \mathcal{P}\left(\mathbf{w}_{\mathbf{k}_{1}}\right)
$$




$$
\begin{aligned}
& \propto \int \mathrm{d} \mathbf{w}_{\mathbf{k}_{1}} \delta\left(N r_{\mathbf{k}_{1}}^{2}-\sum_{j \in \mathbb{I}_{P}} w_{\mathbf{k}_{1}, j}^{2}\right) \delta\left(\sum_{j=1}^{N} w_{\mathbf{k}_{1}, j}^{2}-N\right) \\
& \propto \int \mathrm{d} \mathbf{w}_{\mathbf{k}_{1}} \delta\left(N r_{\mathbf{k}_{1}}^{2}-\sum_{j \in \mathbb{I}_{P}} w_{\mathbf{k}_{1}, j}^{2}\right) \delta\left(N r_{\mathbf{k}_{1}}^{2}+\sum_{j \notin \mathbb{I}} w_{\mathbf{k}_{1}, j}^{2}-N\right) \\
& \propto \int \prod_{j \in \mathbb{I}_{P}} \mathrm{~d} w_{\mathbf{k}_{1}, j} \delta\left(\sum_{j \in \mathbb{I}_{P}} w_{\mathbf{k}_{1}, j}^{2}-N r_{\mathbf{k}_{1}}^{2}\right) \int \prod_{j \notin \mathbb{I}_{P}} \mathrm{~d} w_{\mathbf{k}_{1}, j} \delta\left(\sum_{j \notin \mathbb{I}_{P}} w_{\mathbf{k}_{1}, j}^{2}-N\left(1-r_{\mathbf{k}_{1}}^{2}\right)\right) \\
& \propto r_{\mathbf{k}_{1}}^{P-1}\left(1-r_{\mathbf{k}_{1}}^{2}\right) \\
& =\left[N B\left(\frac{P+1}{2}, \frac{N-P+1) / 2}{2}\right)\right]^{-1} r_{\mathbf{k}_{1}}^{P-1}\left(1-r_{\mathbf{k}_{1}}^{2}\right)^{(N-P-1) / 2},
\end{aligned}
$$

where $B(x, y)=\Gamma(x) \Gamma(y) / \Gamma(x+y)$ is the beta function. For the off-diagonal elements we proceed in the same manner

$$
\begin{aligned}
\mathcal{P}\left(r_{\mathbf{k}_{1}} r_{\mathbf{k}_{1}^{\prime}}\right. & \left.\cos \left(\varphi_{\mathbf{k}_{1} \mathbf{k}_{1}^{\prime}}\right)\right)=\int \mathrm{d} \mathbf{w}_{\mathbf{k}_{1}} \mathrm{~d} \mathbf{w}_{\mathbf{k}_{1}^{\prime}} \mathcal{P}\left(r_{\mathbf{k}_{1}} r_{\mathbf{k}_{1}^{\prime}} \cos \left(\varphi_{\mathbf{k}_{1} \mathbf{k}_{1}^{\prime}}\right) \mid \mathbf{w}_{\mathbf{k}_{1}}, \mathbf{w}_{\mathbf{k}_{1}^{\prime}}\right) \mathcal{P}\left(\mathbf{w}_{\mathbf{k}_{1}}, \mathbf{w}_{\mathbf{k}_{1}^{\prime}}\right) \\
= & \int \mathrm{d} \mathbf{w}_{\mathbf{k}_{1}} \mathrm{~d} \mathbf{w}_{\mathbf{k}_{1}^{\prime}} \mathcal{P}\left(r_{\mathbf{k}_{1}} r_{\mathbf{k}_{1}^{\prime}} \cos \left(\varphi_{\mathbf{k}_{1} \mathbf{k}_{1}^{\prime}}\right) \mid \mathbf{w}_{\mathbf{k}_{1}}, \mathbf{w}_{\mathbf{k}_{1}^{\prime}}\right) \mathcal{P}\left(\mathbf{w}_{\mathbf{k}_{1}^{\prime}} \mid \mathbf{w}_{\mathbf{k}_{1}}\right) \mathcal{P}\left(\mathbf{w}_{\mathbf{k}_{1}}\right) \\
\propto & \int \mathrm{d} \mathbf{w}_{\mathbf{k}_{1}} \mathrm{~d} \mathbf{w}_{\mathbf{k}_{1}^{\prime}} \delta\left(N r_{\mathbf{k}_{1}} r_{\mathbf{k}_{1}^{\prime}} \cos \left(\varphi_{\mathbf{k}_{1} \mathbf{k}_{1}^{\prime}}\right)-\sum_{j \in \mathbb{I}_{P}} w_{\mathbf{k}_{1}, j} w_{\mathbf{k}_{1}^{\prime}, j}\right) \delta\left(N r_{\mathbf{k}_{1}}^{2}-\sum_{j \in \mathbb{I}_{P}} w_{\mathbf{k}_{1}, j}^{2}\right) \\
& \times \delta\left(N r_{\mathbf{k}_{1}^{\prime}}^{2}-\sum_{j \in \mathbb{I}_{P}} w_{\mathbf{k}_{1}^{\prime}, j}^{2}\right) \delta\left(\sum_{j=1}^{N} w_{\mathbf{k}_{1}, j} w_{\mathbf{k}_{1}^{\prime}, j}-N \tilde{\zeta}_{\mathbf{k}_{1} \mathbf{k}_{1}^{\prime}}\right) \\
& \times \delta\left(\sum_{j=1}^{N} w_{\mathbf{k}_{1}, j}^{2}-N\right) \delta\left(\sum_{j=1}^{N} w_{\mathbf{k}_{1}^{\prime}, j}^{2}-N\right)
\end{aligned}
$$

where $\tilde{\zeta}_{\mathbf{k}_{1} \mathbf{k}_{1}^{\prime}}$ is the overlap between the vectors $\mathbf{w}_{\mathbf{k}_{1}}$ and $\mathbf{w}_{\mathbf{k}_{1}^{\prime}}$. Observe that as in the case of the diagonal elements we can separate the variables into two groups, according to whether their indexes are in $\mathbb{I}_{P}$ or not. Thus

$$
\begin{aligned}
& \mathcal{P}\left(r_{\mathbf{k}_{1}} r_{\mathbf{k}_{1}^{\prime}}\right.\left.\cos \left(\varphi_{\mathbf{k}_{1} \mathbf{k}_{1}^{\prime}}\right)\right)=\int \prod_{j \in \mathbb{I}_{P}} \mathrm{~d} w_{\mathbf{k}_{1}, j} \mathrm{~d} w_{\mathbf{k}_{1}^{\prime}, j} \delta\left(N r_{\mathbf{k}_{1}}^{2}-\sum_{j \in \mathbb{I}_{P}} w_{\mathbf{k}_{1}, j}^{2}\right) \delta\left(N r_{\mathbf{k}_{1}^{\prime}}^{2}-\sum_{j \in \mathbb{I}_{P}} w_{\mathbf{k}_{1}^{\prime}, j}^{2}\right) \\
& \times \delta\left(N r_{\mathbf{k}_{1}} r_{\mathbf{k}_{1}^{\prime}} \cos \left(\varphi_{\mathbf{k}_{1} \mathbf{k}_{1}^{\prime}}\right)-\sum_{j \in \mathbb{I}_{P}} w_{\mathbf{k}_{1}, j} w_{\mathbf{k}_{1}^{\prime}, j}\right) \\
& \times \int \prod_{j \notin \mathbb{I}_{P}} \mathrm{~d} w_{\mathbf{k}_{1}, j} \mathrm{~d} w_{\mathbf{k}_{1}^{\prime}, j} \delta\left(\sum_{j \notin \mathbb{I}_{P}} w_{\mathbf{k}_{1}, j}^{2}-N\left(1-r_{\mathbf{k}_{1}}^{2}\right)\right) \delta\left(\sum_{j \notin \mathbb{I}_{P}} w_{\mathbf{k}_{1}^{\prime}, j}^{2}-N\left(1-r_{\mathbf{k}_{1}^{\prime}}^{2}\right)\right) \\
& \times \delta\left(\sum_{j \notin \mathbb{I}_{P}} w_{\mathbf{k}_{1}, j} w_{\mathbf{k}_{1}^{\prime}, j}-N\left(\tilde{\zeta}_{\mathbf{k}_{1} \mathbf{k}_{1}^{\prime}}-r_{\mathbf{k}_{1}} r_{\mathbf{k}_{1}^{\prime}} \cos \left(\varphi_{\mathbf{k}_{1} \mathbf{k}_{1}^{\prime}}\right)\right)\right) .
\end{aligned}
$$


Let us define the overlap $\cos \left(\tilde{\varphi}_{\mathbf{k}_{1} \mathbf{k}_{1}^{\prime}}\right)$ by

$$
\cos \left(\tilde{\varphi}_{\mathbf{k}_{1} \mathbf{k}_{1}^{\prime}}\right) \equiv \frac{\tilde{\zeta}_{\mathbf{k}_{1} \mathbf{k}_{1}^{\prime}}-r_{\mathbf{k}_{1}} r_{\mathbf{k}_{1}^{\prime}} \cos \left(\varphi_{\mathbf{k}_{1} \mathbf{k}_{1}^{\prime}}\right)}{\sqrt{\left(1-r_{\mathbf{k}_{1}}^{2}\right)\left(1-r_{\mathbf{k}_{1}^{\prime}}^{2}\right)}} .
$$

The integrals to be solved are of the form

$$
\mathcal{I} \equiv \int_{\mathbb{R}^{d}} \mathrm{~d} \boldsymbol{x}_{1} \mathrm{~d} \boldsymbol{x}_{2} \delta\left(\boldsymbol{x}_{1} \cdot \boldsymbol{x}_{2}-N r_{1} r_{2} \cos (\varphi)\right) \delta\left(\left|\boldsymbol{x}_{1}\right|^{2}-N r_{1}^{2}\right) \delta\left(\left|\boldsymbol{x}_{2}\right|^{2}-N r_{2}^{2}\right)
$$

which is proportional to the length of the hyper-ring around $\boldsymbol{x}_{2}$ with radius $\left|\boldsymbol{x}_{1}\right| \sin (\varphi)$ times the length of the hyper-ring around $\boldsymbol{x}_{1}$ with radius $\left|\boldsymbol{x}_{2}\right| \sin (\varphi)$. Therefore

$$
\mathcal{I} \propto\left(r_{1} r_{2}\right)^{d-1} \sin ^{d-2}(\varphi)
$$

thus

$\mathcal{P}\left(r_{\mathbf{k}_{1}} r_{\mathbf{k}_{1}^{\prime}} \cos \left(\varphi_{\mathbf{k}_{1} \mathbf{k}_{1}^{\prime}}\right)\right) \propto\left(r_{\mathbf{k}_{1}} r_{\mathbf{k}_{1}^{\prime}}\right)^{P-1} \sin ^{P-2}\left(\varphi_{\mathbf{k}_{1} \mathbf{k}_{1}^{\prime}}\right)\left[\left(1-r_{\mathbf{k}_{1}}^{2}\right)\left(1-r_{\mathbf{k}_{1}^{\prime}}^{2}\right)\right]^{(N-P-1) / 2} \sin ^{N-P-2}\left(\tilde{\varphi}_{\mathbf{k}_{1} \mathbf{k}_{1}^{\prime}}\right)$,

and, by (B.1) we have that

$\left(1-r_{\mathbf{k}_{1}}^{2}\right)\left(1-r_{\mathbf{k}_{1}^{\prime}}^{2}\right) \sin ^{2}\left(\tilde{\varphi}_{\mathbf{k}_{1} \mathbf{k}_{1}^{\prime}}\right)=\left(1-\tilde{\zeta}_{\mathbf{k}_{1} \mathbf{k}_{1}^{\prime}}^{2}\right)-r_{\mathbf{k}_{1}}^{2}-r_{\mathbf{k}_{1}^{\prime}}^{2}+2 r_{\mathbf{k}_{1}} r_{\mathbf{k}_{1}^{\prime}} \tilde{\zeta}_{\mathbf{k}_{1} \mathbf{k}_{1}^{\prime}} \cos \left(\varphi_{\mathbf{k}_{1} \mathbf{k}_{1}^{\prime}}\right)+r_{\mathbf{k}_{1}} r_{\mathbf{k}_{1}^{\prime}} \sin ^{2}\left(\varphi_{\mathbf{k}_{1} \mathbf{k}_{1}^{\prime}}\right)$.

Therefore

$$
\begin{aligned}
& \mathcal{P}\left(r_{\mathbf{k}_{1}}^{2}\right) \propto r_{\mathbf{k}_{1}}^{P-1}\left(1-r_{\mathbf{k}_{1}}^{2}\right)^{(N-P-1) / 2} \\
& \mathcal{P}\left(r_{\mathbf{k}_{1}} r_{\mathbf{k}_{1}^{\prime}} \cos \left(\varphi_{\mathbf{k}_{1} \mathbf{k}_{1}^{\prime}}\right)\right) \propto\left(r_{\mathbf{k}_{1}} r_{\mathbf{k}_{1}^{\prime}}\right)^{P-1} \sin ^{P-2}\left(\varphi_{\mathbf{k}_{1} \mathbf{k}_{1}^{\prime}}\right) \sqrt{\left(1-r_{\mathbf{k}_{1}}^{2}\right)\left(1-r_{\mathbf{k}_{1}^{\prime}}^{2}\right)} \\
& \quad\left[1-\tilde{\zeta}_{\mathbf{k}_{1} \mathbf{k}_{1}^{\prime}}^{2}-r_{\mathbf{k}_{1}}^{2}-r_{\mathbf{k}_{1}^{\prime}}^{2}+2 r_{\mathbf{k}_{1}} r_{\mathbf{k}_{1}^{\prime}} \tilde{\zeta}_{\mathbf{k}_{1} \mathbf{k}_{1}^{\prime}} \cos \left(\varphi_{\mathbf{k}_{1} \mathbf{k}_{1}^{\prime}}\right)+r_{\mathbf{k}_{1}}^{2} r_{\mathbf{k}_{1}^{\prime}}^{2} \sin ^{2}\left(\varphi_{\mathbf{k}_{1} \mathbf{k}_{1}^{\prime}}\right)\right]^{(N-P-2) / 2} .
\end{aligned}
$$

In the thermodynamic limit, where $P \leq N \rightarrow \infty$ and $P / N=p$, the saddle point equations of the Laplace's method are

$$
0=\frac{p}{r_{\mathbf{k}_{1}}}-\frac{1-p}{1-r_{\mathbf{k}_{1}}^{2}} r_{\mathbf{k}_{1}} \quad \forall \mathbf{k}_{1}
$$

for the diagonal elements, and

$$
\begin{array}{rl}
0= & \frac{p}{r_{\mathbf{k}_{1}}}-\frac{1-p}{2} \frac{2 r_{\mathbf{k}_{1}}-2 r_{\mathbf{k}_{1}^{\prime}} \tilde{\zeta}_{\mathbf{k}_{1} \mathbf{k}_{1}^{\prime}} \cos \left(\varphi_{\mathbf{k}_{1} \mathbf{k}_{1}^{\prime}}\right)-2 r_{\mathbf{k}_{1}} r_{\mathbf{k}_{1}^{\prime}}^{2} \sin ^{2}\left(\varphi_{\mathbf{k}_{1} \mathbf{k}_{1}^{\prime}}\right)}{1-\tilde{\zeta}_{\mathbf{k}_{1} \mathbf{k}_{1}^{\prime}}^{2}-r_{\mathbf{k}_{1}}^{2}-r_{\mathbf{k}_{1}^{\prime}}^{2}+2 r_{\mathbf{k}_{1}} r_{\mathbf{k}_{1}^{\prime}} \tilde{\zeta}_{\mathbf{k}_{1} \mathbf{k}_{1}^{\prime}} \cos \left(\varphi_{\mathbf{k}_{1} \mathbf{k}_{1}^{\prime}}\right)+r_{\mathbf{k}_{1}}^{2} r_{\mathbf{k}_{1}^{\prime}}^{2} \sin ^{2}\left(\varphi_{\mathbf{k}_{1} \mathbf{k}_{1}^{\prime}}\right)}(\mathrm{B} \\
0= & \frac{p}{r_{\mathbf{k}_{1}^{\prime}}}-\frac{1-p}{2} \frac{2 r_{\mathbf{k}_{1}^{\prime}}-2 r_{\mathbf{k}_{1}} \tilde{\zeta}_{\mathbf{k}_{1} \mathbf{k}_{1}^{\prime}} \cos \left(\varphi_{\mathbf{k}_{1} \mathbf{k}_{1}^{\prime}}\right)-2 r_{\mathbf{k}_{1}}^{2} r_{\mathbf{k}_{1}^{\prime}} \sin ^{2}\left(\varphi_{\mathbf{k}_{1} \mathbf{k}_{1}^{\prime}}\right)}{1-\tilde{\zeta}_{\mathbf{k}_{1} \mathbf{k}_{1}^{\prime}}^{2}-r_{\mathbf{k}_{1}}^{2}-r_{\mathbf{k}_{1}^{\prime}}^{2}+2 r_{\mathbf{k}_{1}} r_{\mathbf{k}_{1}^{\prime}} \tilde{\zeta}_{\mathbf{k}_{1} \mathbf{k}_{1}^{\prime}} \cos \left(\varphi_{\mathbf{k}_{1} \mathbf{k}_{1}^{\prime}}\right)+r_{\mathbf{k}_{1}}^{2} r_{\mathbf{k}_{1}^{\prime}}^{2} \sin ^{2}\left(\varphi_{\mathbf{k}_{1} \mathbf{k}_{1}^{\prime}}\right)}(\mathrm{B} \\
0 & p \operatorname{cotg}\left(\varphi_{\mathbf{k}_{1} \mathbf{k}_{1}^{\prime}}\right) \\
& -\frac{1-p}{2} \frac{2 r_{\mathbf{k}_{1}} r_{\mathbf{k}_{1}^{\prime}} \tilde{\zeta}_{\mathbf{k}_{1} \mathbf{k}_{1}^{\prime}} \sin \left(\varphi_{\mathbf{k}_{1} \mathbf{k}_{1}^{\prime}}\right)-2 r_{\mathbf{k}_{1}}^{2} r_{\mathbf{k}_{1}^{\prime}}^{2} \cos \left(\varphi_{\mathbf{k}_{1} \mathbf{k}_{1}^{\prime}}\right) \sin \left(\varphi_{\mathbf{k}_{1} \mathbf{k}_{1}^{\prime}}\right)}{1-\tilde{\zeta}_{\mathbf{k}_{1} \mathbf{k}_{1}^{\prime}}^{2}-r_{\mathbf{k}_{1}}^{2}-r_{\mathbf{k}_{1}^{\prime}}^{2}+2 r_{\mathbf{k}_{1}} r_{\mathbf{k}_{1}} \tilde{\zeta}_{\mathbf{k}_{1} \mathbf{k}_{1}^{\prime}} \cos \left(\varphi_{\mathbf{k}_{1} \mathbf{k}_{1}^{\prime}}\right)+r_{\mathbf{k}_{1}}^{2} r_{\mathbf{k}_{1}^{\prime}}^{2} \sin ^{2}\left(\varphi_{\mathbf{k}_{1} \mathbf{k}_{1}^{\prime}}\right)},
\end{array}
$$

$\forall \mathbf{k}_{1} \neq \mathbf{k}_{1}^{\prime}$. From (B.2) we have that $r_{\mathbf{k}_{1}}^{2}=p \forall \mathbf{k}_{1}$. Equations (B.3) and (B.4) are identical, therefore we can suppose that in the limit $r_{\mathbf{k}_{1}}=r_{\mathbf{k}_{1}^{\prime}}=r$ and the system gets reduced 
to only two equations

$$
\begin{aligned}
& 0=p-(1-p) r^{2} \frac{1-\tilde{\zeta}_{\mathbf{k}_{1} \mathbf{k}_{1}^{\prime}} \cos \left(\varphi_{\mathbf{k}_{1} \mathbf{k}_{1}^{\prime}}\right)-r^{2} \sin ^{2}\left(\varphi_{\mathbf{k}_{1} \mathbf{k}_{1}^{\prime}}\right)}{1-\tilde{\zeta}_{\mathbf{k}_{1} \mathbf{k}_{1}^{\prime}}^{2}-2 r^{2}\left(1-\tilde{\zeta}_{\mathbf{k}_{1} \mathbf{k}_{1}^{\prime}} \cos \left(\varphi_{\mathbf{k}_{1} \mathbf{k}_{1}^{\prime}}\right)\right)+r^{4} \sin ^{2}\left(\varphi_{\mathbf{k}_{1} \mathbf{k}_{1}^{\prime}}\right)} \\
& 0=p \operatorname{cotg}\left(\varphi_{\mathbf{k}_{1} \mathbf{k}_{1}^{\prime}}\right)-(1-p) r^{2} \frac{\tilde{\zeta}_{\mathbf{k}_{1} \mathbf{k}_{1}^{\prime}} \sin \left(\varphi_{\mathbf{k}_{1} \mathbf{k}_{1}^{\prime}}\right)-r^{2} \cos \left(\varphi_{\mathbf{k}_{1} \mathbf{k}_{1}^{\prime}}\right) \sin \left(\varphi_{\mathbf{k}_{1} \mathbf{k}_{1}^{\prime}}\right)}{1-\tilde{\zeta}_{\mathbf{k}_{1} \mathbf{k}_{1}^{\prime}}^{2}-2 r^{2}\left(1-\tilde{\zeta}_{\mathbf{k}_{1} \mathbf{k}_{1}^{\prime}} \cos \left(\varphi_{\mathbf{k}_{1} \mathbf{k}_{1}^{\prime}}\right)\right)+r^{4} \sin ^{2}\left(\varphi_{\mathbf{k}_{1} \mathbf{k}_{1}^{\prime}}\right)}
\end{aligned}
$$

which accepts the solution $r^{2}=p$ and $\cos \left(\varphi_{\mathbf{k}_{1} \mathbf{k}_{1}^{\prime}}\right)=\tilde{\zeta}_{\mathbf{k}_{1} \mathbf{k}_{1}^{\prime}} \forall \mathbf{k}_{1} \mathbf{k}_{1}^{\prime}$. Therefore, in the thermodynamic limit, we have that

$$
\begin{array}{ll}
\mathcal{P}\left(r_{\mathbf{k}_{1}}^{2}\right) & \propto \delta\left(r_{\mathbf{k}_{1}}^{2}-p\right) \\
\mathcal{P}\left(r_{\mathbf{k}_{1}} r_{\mathbf{k}_{1}^{\prime}} \cos \left(\varphi_{\mathbf{k}_{1} \mathbf{k}_{1}^{\prime}}\right)\right) & \propto \delta\left(r_{\mathbf{k}_{1}}^{2}-p\right) \delta\left(r_{\mathbf{k}_{1}^{\prime}}^{2}-p\right) \delta\left(\cos \left(\varphi_{\mathbf{k}_{1} \mathbf{k}_{1}^{\prime}}\right)-\tilde{\zeta}_{\mathbf{k}_{1} \mathbf{k}_{1}^{\prime}}\right)
\end{array}
$$

which implies that the expected value of the matrix of partial overlaps given these distributions of elements, is

$$
\lim _{P \leq N \rightarrow \infty}\left(\begin{array}{l}
N \\
P
\end{array}\right)^{-1} \sum_{\mathbb{I}_{P}} \frac{1}{N} \sum_{j \in \mathbb{I}_{P}} w_{\mathbf{k}_{1}, j} w_{\mathbf{k}_{1}^{\prime}, j}=p[\boldsymbol{Q}]_{\mathbf{k}_{1}, \mathbf{k}_{1}^{\prime}} .
$$

\section{Appendix C. Integrals}

In here we present the calculation of several integrals that appear in the main development.

Appendix C.1. Integrals related to (A.12)

In order to simplify the following developments we will suppose that $\exists M \in \mathbb{N} \mid K=$ $2 M+1$. The integrals to be solved are

$$
\begin{aligned}
b_{0}^{M} & \equiv \int \mathcal{D}(\eta, \hat{\eta}) \cos (\hat{\eta})^{2 M+1} \\
b_{m}^{M} & \equiv \int \mathcal{D}(\eta, \hat{\eta}) \cos (\hat{\eta})^{2(M-m)+1} \sin (\hat{\eta})^{2 m} \\
a_{m}^{M} & \equiv \int \mathcal{D}(\eta, \hat{\eta}) \cos (\hat{\eta})^{2(M-m)} \sin (\hat{\eta})^{2 m+1}
\end{aligned}
$$

Before computing the integrals observe that for all $A>0$ and $B \geq 0$

$$
\begin{aligned}
\int \mathcal{D}(\eta, \hat{\eta}) \sin (A \hat{\eta}) & =-\frac{i}{4 \pi} \int_{0}^{\infty} \mathrm{d} \eta \int_{-\infty}^{\infty} \mathrm{d} \hat{\eta} \exp (-i \hat{\eta} \eta)[\exp (i \hat{\eta} A)-\exp (-i \hat{\eta} A)] \\
& =-\frac{i}{4 \pi} \int_{0}^{\infty} \mathrm{d} \eta \int_{-\infty}^{\infty} \mathrm{d} \hat{\eta}[\exp [-i \hat{\eta}(\eta-A)]-\exp [-i \hat{\eta}(\eta+A)]] \\
& =-\frac{i}{2}[\Theta(A)-\Theta(-A)] \\
& =-\frac{i}{2}
\end{aligned}
$$


similarly

$$
\begin{aligned}
\int \mathcal{D}(\eta, \hat{\eta}) \cos (A \hat{\eta}) \cos (B \hat{\eta}) & =\frac{1}{4}[\Theta(A+B)+\Theta(-A-B)+\Theta(A-B)+\Theta(-A+B)] \\
& =\frac{1}{2}
\end{aligned}
$$

and

$$
\begin{aligned}
\int \mathcal{D}(\eta, \hat{\eta}) \cos (A \hat{\eta}) \sin (B \hat{\eta}) & =-\frac{i}{4}[\Theta(A+B)-\Theta(A-B)+\Theta(-A+B)-\Theta(-A-B)] \\
& =-\frac{i}{2} \Theta(B-A) .
\end{aligned}
$$

The first integral is

$$
\begin{aligned}
b_{0}^{M} & =\int \mathcal{D}(\eta, \hat{\eta}) \cos (\hat{\eta})^{2 M+1}=\frac{1}{2^{2 M}} \sum_{k=0}^{M}\left(\begin{array}{c}
2 M+1 \\
k
\end{array}\right) \int \mathcal{D}(\eta, \hat{\eta}) \cos [(2 M-2 k+1) \hat{\eta}] \\
& =\frac{1}{2^{2 M+1}} \sum_{k=0}^{M}\left(\begin{array}{c}
2 M+1 \\
k
\end{array}\right)=\frac{1}{2} \quad \forall M .
\end{aligned}
$$

The second integral is

$$
\begin{aligned}
b_{m}^{M}= & \int \mathcal{D}(\eta, \hat{\eta}) \cos (\hat{\eta})^{2(M-m)+1} \sin (\hat{\eta})^{2 m} \\
= & \int \mathcal{D}(\eta, \hat{\eta}) \frac{1}{2^{2(M-m)}} \sum_{k=0}^{M-m}\left(\begin{array}{c}
2(M-m)+1 \\
k
\end{array}\right) \cos [(2 M-2(k+m)+1) \hat{\eta}] \\
& \frac{1}{2^{2 m}}\left\{2 \sum_{j=0}^{m-1}(-1)^{m-j}\left(\begin{array}{c}
2 m \\
j
\end{array}\right) \cos [(2 m-2 j) \hat{\eta}]+\left(\begin{array}{c}
2 m \\
m
\end{array}\right)\right\} \\
= & \frac{1}{2^{2 M}} \sum_{k=0}^{M-m}\left(\begin{array}{c}
2(M-m)+1 \\
k
\end{array}\right) \sum_{j=0}^{m-1}(-1)^{m-j}\left(\begin{array}{c}
2 m \\
j
\end{array}\right)+\frac{1}{2^{2 m+1}}\left(\begin{array}{c}
2 m \\
m
\end{array}\right) \\
= & \frac{1}{2^{2 M}} \frac{2^{2(M-m)+1}}{2}\left[\begin{array}{c}
1 \\
2
\end{array}\left(\begin{array}{c}
2 m \\
m
\end{array}\right)\right]+\frac{1}{2^{2 m+1}}\left(\begin{array}{c}
2 m \\
m
\end{array}\right) \\
= & 0 \quad \forall M, m>0 .
\end{aligned}
$$

And the last integral is then

$$
\begin{aligned}
a_{m}^{M}= & \int \mathcal{D}(\eta, \hat{\eta}) \cos (\hat{\eta})^{2(M-m)} \sin (\hat{\eta})^{2 m+1} \\
= & \int \mathcal{D}(\eta, \hat{\eta}) \cos (\hat{\eta})^{2(M-m)}\left[1-\cos ^{2}(\hat{\eta})\right]^{m} \sin (\hat{\eta}) \\
= & \int \mathcal{D}(\eta, \hat{\eta}) \sum_{\ell=0}^{m}(-1)^{\ell}\left(\begin{array}{c}
m \\
\ell
\end{array}\right) \cos (\hat{\eta})^{2(M-m+\ell)} \sin (\hat{\eta}) \\
= & \sum_{\ell=0}^{m}(-1)^{\ell}\left(\begin{array}{c}
m \\
\ell
\end{array}\right) \frac{(-i)}{2^{2(M-m+\ell)}}\left\{\frac{1}{2}\left(\begin{array}{c}
2(M-m+\ell) \\
M-m+\ell
\end{array}\right)+\right. \\
& \left.\quad+\sum_{k=0}^{M-m+\ell-1}\left(\begin{array}{c}
2(M-m+\ell) \\
k
\end{array}\right) \Theta[1-2(M-m+\ell-k)]\right\}
\end{aligned}
$$




$$
\begin{aligned}
& =-\frac{i}{2^{2(M-m)+1}} \sum_{\ell=0}^{m}\left(-\frac{1}{4}\right)^{\ell}\left(\begin{array}{c}
m \\
\ell
\end{array}\right)\left(\begin{array}{c}
2(M-m+\ell) \\
M-m+\ell
\end{array}\right) \\
& =-\frac{i}{2^{2 M+1}}\left(\begin{array}{c}
M \\
m
\end{array}\right)^{-1}\left(\begin{array}{c}
2 m \\
m
\end{array}\right)\left(\begin{array}{c}
2(M-m) \\
M-m
\end{array}\right) .
\end{aligned}
$$

\section{Appendix D. Derivation of condition (10)}

We start by writing the average discrepancy component as

$$
\mathfrak{d}_{P}^{(N)}[f]=\frac{1}{2}-\frac{1}{2} \sum_{\{\mathbf{S}\},\left\{\mathbf{S}^{\prime}\right\}} \mathcal{P}\left(\mathbf{S}, \mathbf{S}^{\prime}\right) f(\mathbf{S}) f\left(\mathbf{S}^{\prime}\right)
$$

where $\mathcal{P}\left(\mathbf{S}, \mathbf{S}^{\prime}\right)=2^{-N}\left(\begin{array}{l}N \\ P\end{array}\right)^{-1} \delta_{\mathbf{S}^{\mathrm{T}} \mathbf{S}^{\prime}, N-2 P}$. The second term can be then expressed as

$$
\sum_{\{\mathbf{S}\},\left\{\mathbf{S}^{\prime}\right\}} \mathcal{P}\left(\mathbf{S}, \mathbf{S}^{\prime}\right) f(\mathbf{S}) f\left(\mathbf{S}^{\prime}\right)=2^{-N}\left(\begin{array}{c}
N \\
P
\end{array}\right)^{-1} \sum_{\{\mathbf{S}\},\left\{\mathbf{S}^{\prime}\right\}} \delta_{\mathbf{S}^{\top} \mathbf{S}^{\prime}, N-2 P} \sum_{\mathbb{I}, \mathbb{I}} \hat{f}_{\mathbb{I}} \hat{f}_{\mathbb{J}} \prod_{k \in \mathbb{I}} \prod_{l \in \mathbb{I}} S_{k} S_{l}^{\prime}
$$

By expressing the Kronecker delta as

$$
\delta_{r, m}=\frac{1}{2 \pi i} \oint \frac{\mathrm{d} Z}{Z^{m+1}} Z^{r}
$$

we have

$$
\begin{aligned}
& \sum_{\{\mathbf{S}\},\left\{\mathbf{S}^{\prime}\right\}} \mathcal{P}\left(\mathbf{S}, \mathbf{S}^{\prime}\right) f(\mathbf{S}) f\left(\mathbf{S}^{\prime}\right)= \\
& 2^{-N}\left(\begin{array}{c}
N \\
P
\end{array}\right)^{-1} \sum_{\mathbb{I}, \mathbb{J}} \hat{f}_{\mathbb{I}} \hat{f}_{\mathbb{J}} \frac{1}{2 \pi i} \oint \frac{\mathrm{d} Z}{Z^{P+1}} \prod_{k \in \mathbb{I}} \prod_{l \in \mathbb{J}} \prod_{j=1}^{N} \frac{1}{2} \sum_{S_{j}= \pm 1} \sum_{S_{j}^{\prime}= \pm 1} Z^{\frac{1}{2}\left(1-S_{j} S_{j}^{\prime}\right)} S_{k} S_{l}^{\prime} .
\end{aligned}
$$

Now we have to take the average over the input variables. To do so we define the following sets: $\mathbb{A} \equiv \mathbb{I} \cap \mathbb{J}, \mathbb{B} \equiv \mathbb{I} / \mathbb{A}, \mathbb{C} \equiv \mathbb{J} / \mathbb{A}$ and $\mathbb{D} \equiv[N] /(\mathbb{I} \cup \mathbb{J})$. These four sets completely cover $[N]$ without intersecting each other. The spin average is then

$$
\begin{aligned}
& g(Z) \equiv \prod_{j=1}^{N} \frac{1}{2} \sum_{S_{j}= \pm 1} \sum_{S_{j}^{\prime}= \pm 1} Z^{\frac{1}{2}\left(1-S_{j} S_{j}^{\prime}\right)} \prod_{k \in \mathbb{I}} S_{k} \prod_{l \in \mathbb{J}} S_{l}^{\prime} \\
& =(1-Z)^{|\mathbb{A}|} \delta_{\mathbb{B}, \varnothing} \delta_{\mathbb{C}, \varnothing}(1+Z)^{|\mathbb{D}|} .
\end{aligned}
$$

If $\mathbb{B}=\mathbb{C}=\varnothing$ then $\mathbb{I}=\mathbb{J}$ and then

$$
g(Z)=\delta_{\mathbb{I}, \mathbb{J}}(1-Z)^{|\mathbb{I}|}(1+Z)^{N-|\mathbb{J}|} .
$$

Let us write the sum over the sets of indexes $\sum_{\mathbb{I}}$ as $\sum_{r=1}^{N} \sum_{\mathbb{I}_{r}}$ where $\left|\mathbb{I}_{r}\right|=r$. Then, if

$$
\mathscr{A}_{r} \equiv \sum_{\mathbb{I}_{r}} \hat{f}_{\mathbb{I}_{r}}^{2}
$$

we can write

$$
\mathfrak{d}_{P}^{(N)}[f]=\frac{1}{2}-\frac{1}{2}\left(\begin{array}{l}
N \\
P
\end{array}\right)^{-1} \sum_{r=1}^{N} \mathscr{A}_{r} \frac{1}{2 \pi i} \oint \frac{\mathrm{d} Z}{Z^{P+1}}(1-Z)^{r}(1+Z)^{N-r}
$$




$$
\begin{aligned}
& =\frac{1}{2}-\frac{1}{2}\left(\begin{array}{c}
N \\
P
\end{array}\right)^{-1} \sum_{r=1}^{N} \mathscr{A}_{r} \sum_{j=0}^{r}(-1)^{j}\left(\begin{array}{c}
r \\
j
\end{array}\right) \sum_{k=0}^{N-r}\left(\begin{array}{c}
N-r \\
k
\end{array}\right) \delta_{j+k, P} \\
& =\frac{1}{2}-\frac{1}{2}\left(\begin{array}{c}
N \\
P
\end{array}\right)^{-1} \sum_{r=1}^{N} \mathscr{A}_{r} \sum_{j=0}^{\min \{r, P\}}(-1)^{j}\left(\begin{array}{c}
r \\
j
\end{array}\right)\left(\begin{array}{c}
N-r \\
P-j
\end{array}\right) .
\end{aligned}
$$

In this case the difference of average discrepancy components is

$$
\Delta_{P}^{N}[f] \equiv \mathfrak{d}_{P}^{(N)}[f]-\mathfrak{d}_{P+1}^{(N)}[f]=\frac{1}{2} \sum_{r=1}^{N} \mathscr{A}_{r} \sum_{j=0}^{r}(-1)^{j}\left(\begin{array}{l}
r \\
j
\end{array}\right)\left[\frac{\left(\begin{array}{c}
N-r \\
P-j
\end{array}\right)}{\left(\begin{array}{l}
N \\
P
\end{array}\right)}-\frac{\left(\begin{array}{c}
N-r \\
P+1-j
\end{array}\right)}{\left(\begin{array}{c}
N \\
P+1
\end{array}\right)}\right] .
$$

Observe that

$$
\left(\begin{array}{l}
r \\
j
\end{array}\right)=\frac{\prod_{k=0}^{j-1}(r-k)}{j !}=\frac{\mathrm{P}_{j}(r)}{j !}
$$

where $\mathrm{P}_{j}(r)$ is a polynomial of degree $j$ in $r$. If $P \ll N$ then

$$
\left(\begin{array}{l}
N \\
P
\end{array}\right) \simeq \frac{N^{P}}{P !}+O\left(N^{-1}\right)
$$

Thus

$$
\begin{aligned}
\left(\begin{array}{l}
N \\
P
\end{array}\right)^{-1}\left(\begin{array}{l}
r \\
j
\end{array}\right)\left(\begin{array}{l}
N-r \\
P-j
\end{array}\right) & \simeq\left(\begin{array}{l}
P \\
j
\end{array}\right) \frac{\mathrm{P}_{j}(r) \mathrm{P}_{P-j}(N-r)}{N^{P}}+O\left(N^{-1}\right) \\
& \simeq\left(\begin{array}{c}
P \\
j
\end{array}\right) \frac{a_{P} r^{j}(N-r)^{P-j}}{N^{P}}+O\left(N^{-1}\right)
\end{aligned}
$$

where $a_{P} \in \mathbb{R}$ is a constant. We then have the following expression (disregarding terms of $O(1 / N))$

$$
\begin{aligned}
\Delta_{P}^{N}[f] & \simeq \frac{1}{2} \sum_{r=1}^{N} \mathscr{A}_{r} \\
& \sum_{j=0}^{r}(-1)^{j}\left[\left(\begin{array}{c}
P \\
j
\end{array}\right) a_{P}\left(\frac{r}{N}\right)^{j}\left(1-\frac{r}{N}\right)^{P-j}-\left(\begin{array}{c}
P+1 \\
j
\end{array}\right) a_{P+1}\left(\frac{r}{N}\right)^{j}\left(1-\frac{r}{N}\right)^{P+1-j}\right]
\end{aligned}
$$

and observe that both terms in the sum over $j$ are polynomials of order $P$ and $P+1$ in $r / N$. Thus

$$
\begin{aligned}
\Delta_{P}^{N}[f] & \simeq \frac{1}{2} \sum_{r=1}^{N} \mathscr{A}_{r} \sum_{j=0}^{P+1} b_{j}^{(P)}\left(\frac{r}{N}\right)^{j}+O\left(N^{-1}\right) \\
& \simeq \frac{1}{2} \sum_{j=1}^{P+1} b_{j}^{(P)} \sum_{r=1}^{N} \mathscr{A}_{r}\left(\frac{r}{N}\right)^{j}+O\left(N^{-1}\right)
\end{aligned}
$$

where $b_{j}^{(P)} \in \mathbb{R}$ are the polynomial coefficients and depend only on the value of $P$. From this expression it is clear that in order to satisfy the condition $(7)$ for all values of $P$ we need to require that

$$
\forall j \in \mathbb{N} \lim _{N \rightarrow \infty} \frac{1}{N^{j}} \sum_{r=1}^{N} \mathscr{A}_{r} r^{j}=0,
$$

which is the condition (10) 


\section{Acknowledgments}

The authors acknowledge support from CICYT (Spain) through grant TIN2008-04985 (including FEDER funds) and from Junta de Andalucía through grant P08-TIC-04026.

\section{References}

[1] Franco L and Anthony M 2006 IEEE Trans $N$ N 17578

[2] Neirotti J P 2010 J Phys A 43015101

[3] Neirotti J P 2010 J Phys A 43125101

[4] Cybenko G 1988 Math Control Signals Syst 2303

[5] Engel A 1996 J Phys A 29 L323

[6] Urbanczik R 1997 J Phys A 30 L387

[7] Ahr M Biehl M and Urbanczik R $1999 J$ Phys A 32 L531

[8] Rosen-Zvi M Engel A and Kanter I 2001 Phys Rev Lett 87078101

[9] Schwarze H and Hertz J 1993 J Phys A 264919

[10] Schwarze H 1993 J Phys A 265781

[11] Opper M 1994 Phys Rev Lett 722113

[12] Copelli M and Caticha N 1995 J Phys A 281615

[13] Urbanczik R 1995 J Phys A 287097

[14] Saad D and Solla S A 1995 Phys Rev Lett 744337

[15] Monasson R and Zecchina R 1995 Phys Rev Lett 752432

[16] Vicente R and Caticha N 1997 J Phys A 30 L599

[17] Saad D and Rattray M 1998 Optimal on-line learning in neural networks, On-line learning in neural networks, D Saad ed, Cambridge

[18] Copelli M and Caticha N 1998 Universal asymptotics in committee machines with tree architecture, On-line learning in neural networks, D Saad ed, Cambridge

[19] Kinzel W and Kanter I 2002 Interacting Neural Networks and Cryptography, Advances in Solid State Physics 42, Springer Berlin, Heidelberg

[20] Kurant M and Thiran P 2007 Phys Rev E 76026102

[21] Cousseau F Mimura K and Okada M 2008 Statistical mechanics of lossy compression for nonmonotonic multilayer perceptrons ISIT pg 509, Toronto Canada.

[22] Cousseau F Mimura K and Okada M 2010 Phys Rev E 81021104

[23] Church A 1936 Am J Math 58345

[24] Turing A M 1937 Proc London Math Soc 42230

[25] Hartmanis J and Stearns R E 1965 Trans Am Math Soc 117285

[26] Kolmogorov A N 1965 Problems of Information and Transmission 11

[27] Li M and Vitányi P 1997 An introduction to Kolmogorov Complexity and its Applications, 2nd edition, Springer

[28] Franco L and Cannas S A 2000 Neural Computation 122405

[29] Franco L 2006 Neurocomputing $\mathbf{7 0} 351$

[30] Mezard M Parisi G and Virasoro M A 1987 Spin glasses theory and beyond World Scientific

[31] O'Donnel R W 2003 Computational Applications of Noise Sensitivity PhD Thesis MIT

[32] Stefankovic D 2000 Fourier transform in computer science Master Thesis University of Chicago

[33] Ben-Or M and Linial N 1995 Collective coin flipping (Randomness and Computation Academic Press New York) 